\title{
FATIMA — FAst TIMing Array for DESPEC at FAIR
} \author{
V.F.E. Pucknell ${ }^{\mathrm{f}}$, J.-M. Régis ${ }^{\mathrm{j}}$, H. Schaffner ${ }^{\mathrm{i}}$, J. Simpson ${ }^{\mathrm{f}}$, P. Singh ${ }^{\mathrm{k}}$, C.M. Townsley ${ }^{\mathrm{a}}$, \\ J.F. Smith ${ }^{\mathrm{m}}$, J. Vesic ${ }^{\mathrm{i}, \mathrm{n}}$ \\ ${ }^{a}$ Department of Physics, University of Surrey, Guildford, GU2 7XH, United Kingdom \\ ${ }^{\mathrm{b}}$ Institut für Kernphysik, Technische Universität Darmstadt, Schlossgartenstrasse 9, 64289, Darmstadt, Germany \\ ${ }^{\mathrm{c}}$ National Physical Laboratory, Teddington, Middlesex, TW11 OLW, United Kingdom \\ d School of Computing Engineering and Mathematics, University of Brighton, Brighton BN2 4GJ, United Kingdom \\ ${ }^{\text {e }}$ Faculty of Physics, University of Sofia, 1164 Sofia, Bulgaria \\ ${ }^{\mathrm{f}}$ STFC Daresbury Laboratory, Daresbury, Warrington WA4 4AD, United Kingdom \\ ${ }^{\mathrm{g}}$ Nuclear Physics Group, Schuster Laboratory, University of Manchester, Manchester, M13 9PL, United Kingdom

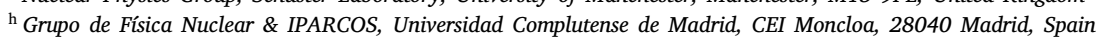 \\ ${ }^{\text {i } G S I ~ H e l m h o l t z z e n t r u m ~ f u ̈ r ~ S c h w e r i o n e n f o r s c h u n g ~ G m b H, ~} 64291$ Darmstadt, Germany \\ j Institut für Kernphysik der Universität zu Köln, Zülpicher Strasse 77, D-50937 Köln, Germany \\ ${ }^{\mathrm{k}}$ Irfu, CEA, Université Paris-Saclay, F-91191 Gif-sur-Yvette, France \\ ${ }^{1}$ Horia Hulubei National Institute of Physics and Nuclear Engineering (IFIN-HH), RO-077125 Bucharest, Romania \\ ${ }^{\mathrm{m}}$ School of Engineering, University of the West of Scotland, Paisley PA1 2BE, United Kingdom \\ ${ }^{\mathrm{n}}$ Jozef Stefan Institute, Jamova cesta 39, 1000 Ljubljana, Slovenia
}

M. Rudigier ${ }^{\mathrm{a}, \mathrm{b}, *}$, Zs. Podolyák ${ }^{\mathrm{a}}$, P.H. Regan ${ }^{\mathrm{a}, \mathrm{c}}$, A.M. Bruce ${ }^{\mathrm{d}}$, S. Lalkovski ${ }^{\mathrm{a}, \mathrm{e}}$, R.L. Canavan ${ }^{\mathrm{a}, \mathrm{c}}$, E.R. Gamba ${ }^{\mathrm{d}}$, O. Roberts ${ }^{\mathrm{d}}$, I. Burrows ${ }^{\mathrm{f}}$, D.M. Cullen ${ }^{\mathrm{g}}$, L.M. Fraile ${ }^{\mathrm{h}}$, L. Gerhard ${ }^{\mathrm{j}}$, J. Gerl ${ }^{\mathrm{i}}$, M. Gorska ${ }^{i}$, A. Grant ${ }^{\mathrm{f}}$, J. Jolie ${ }^{\mathrm{j}}$, V. Karayonchev ${ }^{\mathrm{j}}$, N. Kurz ${ }^{\mathrm{i}}$, W. Korten ${ }^{\mathrm{k}}$, I.H. Lazarus ${ }^{\mathrm{f}}$, C.R. Nita ${ }^{1}$,

\section{A R T I C L E I N F O}

\section{Keywords:}

FAIR

DESPEC

NuSTAR

Fast timing

$\mathrm{LaBr}_{3}(\mathrm{Ce})$ scintillator detectors

\begin{abstract}
A B S T R A C T
The components, working principle and characteristics of FATIMA (FAst TIMing Array), a fast-timing detector system for DESPEC at FAIR, are described. The core system includes $36 \mathrm{LaBr}_{3}(\mathrm{Ce})$ scintillator detectors, a mounting frame for the DESPEC station and a VME-based fast-timing data acquisition system. The current electronic timing circuit is based on V812 constant fraction discriminators and V1290 time-to-digital converters. Gamma-ray energies are measured using V1751 digitisers. Characteristics of the core FATIMA system including efficiency, energy, and coincidence resolving time, as well as limitations, are discussed on the basis of test measurements performed in the S4 cave at GSI, Germany. The coincidence $\gamma-\gamma$ time resolution for the prompt ${ }^{60} \mathrm{Co}$ cascade is determined to be $\sim 320$ ps full width at half maximum. The total full energy peak efficiency at $1 \mathrm{MeV}$ for the 36 detector array in the DESPEC setup is $2.9 \%$. The energy-dependent prompt response centroid curve with the current CFD/TDC combination is shown to be smooth; the centroid shift method can be applied for the measurement of half-lives below 200 ps. An overview of applications of the FATIMA detectors as an ancilliary system in combination with other detector arrays during recent years is given. Data on the operation of the detectors in the presence of magnetic fields are presented.
\end{abstract}

\section{Introduction}

The DESPEC (DEcay SPECtroscopy) experiment [1-3] as part of the NuSTAR project $[4,5]$ for FAIR (Facility for Antiproton and Ion Research) [6] is designed to exploit the new possibilities FAIR will provide in terms of studying exotic nuclides at high beam intensities that are not possible anywhere else. Coupled to the SuperFRS [7], the powerful fragment separator of FAIR, DESPEC will study the decay radiation of implanted ions. The implantation happens in AIDA [8,9], which is a highly segmented active stopper based on Si-strip detectors with a position resolution on the millimetre scale for implantations as well as for subsequent $\beta$ decays or emitted charged particles such as $\alpha$ or protons. Fig. 1 shows a technical drawing of the DESPEC station for FAIR.

Around AIDA, several detector systems can be mounted, according to the experimental needs [10]. FATIMA (FAst TIMing Array) is the detector system of the DESPEC experiment designed to measure lifetimes of excited nuclear states via the method of delayed coincidence electronic fast timing. This experimental technique has come to the

\footnotetext{
* Corresponding author at: Institut für Kernphysik, Technische Universität Darmstadt, Schlossgartenstrasse 9, 64289, Darmstadt, Germany. E-mail address: mrudigier@ikp.tu-darmstadt.de (M. Rudigier).
} 


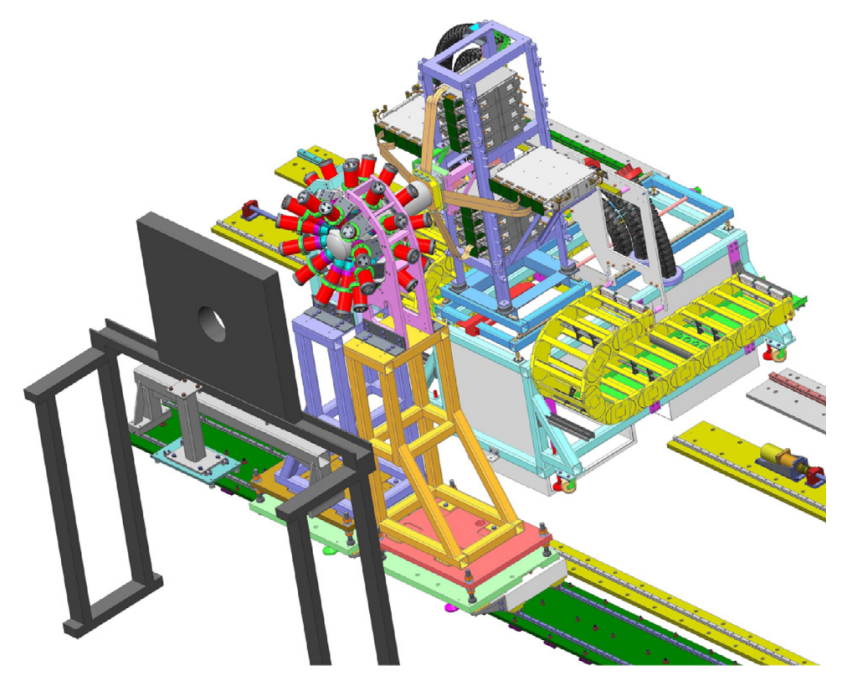

Fig. 1. Technical drawing of the DESPEC setup for FAIR. The FATIMA frame can be seen in the centre with the detectors in red. The beam direction is from the top right (background) to the bottom left (foreground). The same detector mounting is also used for DESPEC experiments at S4 at GSI. The bottom support structure is different due to space restrictions. (For interpretation of the references to colour in this figure legend, the reader is referred to the web version of this article.)

focus in recent years which is emphasised by the fact that similar developments happen at large radioactive ion beam nuclear physics laboratories like MSU/FRIB [11] and TRIUMF [12].

The FATIMA array is made up of $\mathrm{LaBr}_{3}(\mathrm{Ce})$ scintillator detectors. This work is focused on the $36 \mathrm{LaBr}_{3}(\mathrm{Ce})$ detectors which make up the core of the array. Additional rings which will accommodate smaller detectors with different crystal shapes are foreseen to be added [13]. With FATIMA itself, $\gamma-\gamma$ fast timing with few picosecond precision is possible. In combination with a fast plastic detector sandwiched with AIDA, the $\beta-\gamma$ coincidence timing method can be applied, as introduced by Mach et al. [14] and extensively used since the 1990s, e.g. at the ISOLDE facility at CERN [15]. This technique is especially well suited for decay experiments of exotic nuclei, as the efficiency is typically higher than in $\gamma-\gamma$ measurements.

The technical design report for FATIMA was submitted in 2015 [13] to the FAIR Expert Committee Experiments (ECE). Shortly afterwards all components were procured and testing began. By the end of 2015, FATIMA detectors and electronics were used in an experiment at Argonne National Laboratory, together with Digital Gammasphere, to perform decay spectroscopy and lifetime measurements in fission fragments produced in spontaneous fission of ${ }^{252} \mathrm{Cf}$ (see Section 4.1). The detectors have also been used in a number of different experiments in laboratories all around the world. In the summer of 2018 the core FATIMA system was shipped to GSI (Gesellschaft für Schwerionenforschung), Germany - the future site of FAIR. It was integrated with other detectors of the DESPEC setup for the first time in the S4 cave. Most of the data presented in this paper come from test measurements performed during this time.

The features and components of FATIMA are presented in Section 2, and its main characteristics for spectroscopy and timing measurements in Section 3. Section 4 gives some examples of measurements with FATIMA outside the GSI/FAIR scope with a focus on integration of the data acquisition into other systems.

\section{FATIMA detectors and the setup at GSI}

\subsection{Detectors}

The array is made up of 36 congeneric detectors. Each consists of a $\mathrm{LaBr}_{3}(\mathrm{Ce})$ crystal, optically coupled to a photomultiplier tube a)
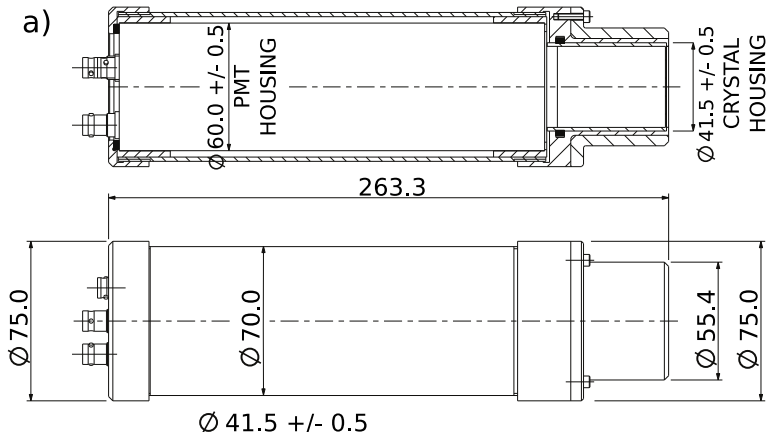

b)

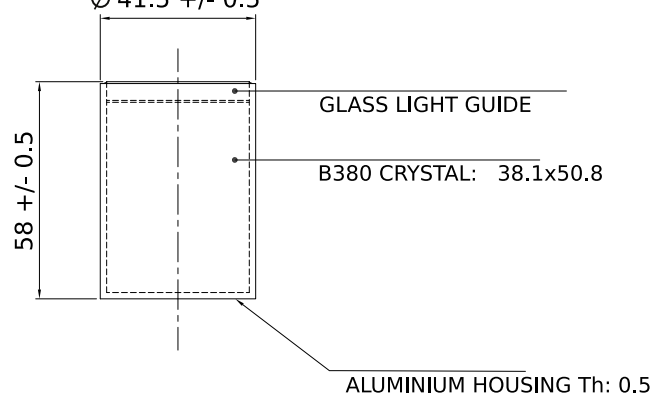

Fig. 2. (a) FATIMA $\mathrm{LaBr}_{3}(\mathrm{Ce})$ detector aluminium case and removable lead shield. (b) Side face drawing of the $\mathrm{LaBr}_{3}(\mathrm{Ce})$ crystal and aluminium housing. The crystal itself measures 1.5 " $\times 2$ " $(38.1 \mathrm{~mm} \times 50.8 \mathrm{~mm})$. Dimensions in the figure are given in $\mathrm{mm}$.

(PMT). These active parts are packed inside an aluminium housing. A photograph of a disassembled detector can be seen in Fig. 3, a drawing of the housing is shown in Fig. 2(a). The crystal can be passively shielded from the sides by attaching a lead cylinder to the front cap. The wall of the lead cylinder is $5 \mathrm{~mm}$ thick.

The crystals were procured from Saint-Gobain, France. The material is $\mathrm{B} 380$, i.e. $\mathrm{LaBr}_{3}(\mathrm{Ce})$ with $5 \% \mathrm{Ce}$ doping. The crystals are cylindrical with a diameter of 1.5 inches and a height of 2 inches as shown in Fig. 2(b). The geometry was chosen based on tests and simulations with different sizes and shapes $[13,16]$. Apart from time and energy resolution considerations, it was also considered that the detectors are able to measure $\gamma$ ray energies up to $4 \mathrm{MeV}$ with reasonable efficiency [16]. In choosing this relatively large size a decision was made to favour increased efficiency per detector, accepting the related decrease in coincidence time resolution (CTR) with increasing crystal size. This was done having in mind the application for experiments in very exotic regions of the nuclear chart. In these experiments, background is usually well controlled by means of particle identification but the actual count rates are rather low (on the order of 10 counts per second in each detector). The reason for not opting for a larger number of smaller detectors, increasing granularity, was mainly the total cost, which scales more strongly with detector number than with crystal size. The FATIMA structure can accommodate two additional rings. The FATIMA detectors for the additional fourth ring have a truncated cone shape [17] and they have been successfully used at ALTO within the $v$-Ball setup (see Section 4). The detectors of these additional rings are not considered in the current work.

As a photomultiplier tube (PMT) the Hamamatsu model H10570 assembly was chosen. The heart of this assembly is the R9779 PMT, featuring eight dynode stages. This choice was made after extensive testing and was based on experience with PMTs for fast timing [13,18, 19] The most important characteristics were linearity of the response and coincidence resolving time.

The detector voltage divider circuit features two output signals, the anode signal and a signal from the eighth dynode (simply called the dynode signal). The negative anode signal is used for timing while the dynode signal is digitised and used to derive information on the detected energy. The maximum voltage to be applied is given as 


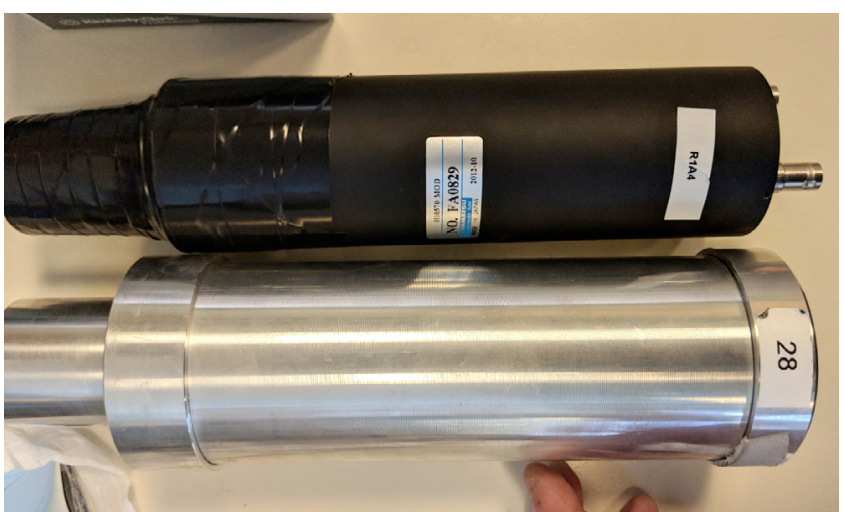

Fig. 3. (Colour online) Picture of the FATIMA detector parts. The PMT can be seen (black tube on top) with a $\mathrm{LaBr}_{3}$ (Ce) crystal taped on. This was how the detectors were mounted for the $v$-Ball experiment at IPN Orsay. The empty aluminium case of this detector can be seen below.

Table 1

List of data acquisition electronics that have been procured for the FATIMA@DESPEC project to date. The list does not include computer workstations to run the DAQ control software (e.g. MBS or MIDAS) or data storage units. All modules are from the manufacturer CAEN.

\begin{tabular}{llll}
\hline Name & Description & Number & $\begin{array}{l}\text { Total number } \\
\text { of channels }\end{array}$ \\
\hline SY4527 & HV control crate & \\
A1535D & HV supply boards & 1 & 36 \\
& VME crate & 2 & \\
V2718 & VME crate controller & 2 & \\
A3818 & Optical controller (PCIx card) & 2 & 40 \\
V1751-DPP-PSD & VME Digitiser & 5 & 48 \\
V812 & VME CFD & 3 & 64 \\
V1290 & VME TDC & 2 & \\
V1495 & VME logic module & 1 & \\
\hline
\end{tabular}

$-1750 \mathrm{~V}$ by Hamamatsu. For this voltage an average current of $385 \mu \mathrm{A}$ is to be expected. During the measurements presented in this work the voltage was typically between -1000 and $-1200 \mathrm{~V}$. The corresponding currents observed were between 280 and $300 \mu \mathrm{A}$.

The material used to produce the $\operatorname{LaBr}_{3}(\mathrm{Ce})$ crystal contains the long lived radioactive isotopes ${ }^{138} \mathrm{La}$ and ${ }^{227} \mathrm{Ac}$. The latter is a chemical homologue to La and is left in the material after chemical purification. Decay radiation from these isotopes is an inherent source of background when using these detectors. This is called the internal activity and a detailed account of this can be found in [20].

\subsection{Mechanical components}

The system is designed to work as part of the DESPEC station of FAIR in combination with AIDA [9] or a similar position sensitive implantation device, and other detector systems, like DEGAS [21]. The FATIMA mounting structure for this purpose was designed and built at STFC Daresbury Laboratory, UK. The structure can currently accommodate 36 detectors, arranged in three rings of 12 detectors each. This constitutes the FATIMA core system. Fig. 4 shows a drawing of one half of the setup. The front face of each detector is tangent to a sphere around the focus point. The rings are situated at $44^{\circ},-6^{\circ}$, and $-44^{\circ}$ with respect to a plane orthogonal to the beam direction. This arrangement was chosen based on simulations which estimated the efficiency [16]. The middle ring is offset from $0^{\circ}$ to avoid the "shadow" of the implantation array or target frame in the centre of the instrument (e.g. AIDA).

A drawing of the FATIMA frame for the DESPEC setup is shown in Fig. 1. Photographs of the FATIMA frame with three fully equipped rings, installed in the S4 cave at GSI, are shown in Fig. 14(g) and (h) in

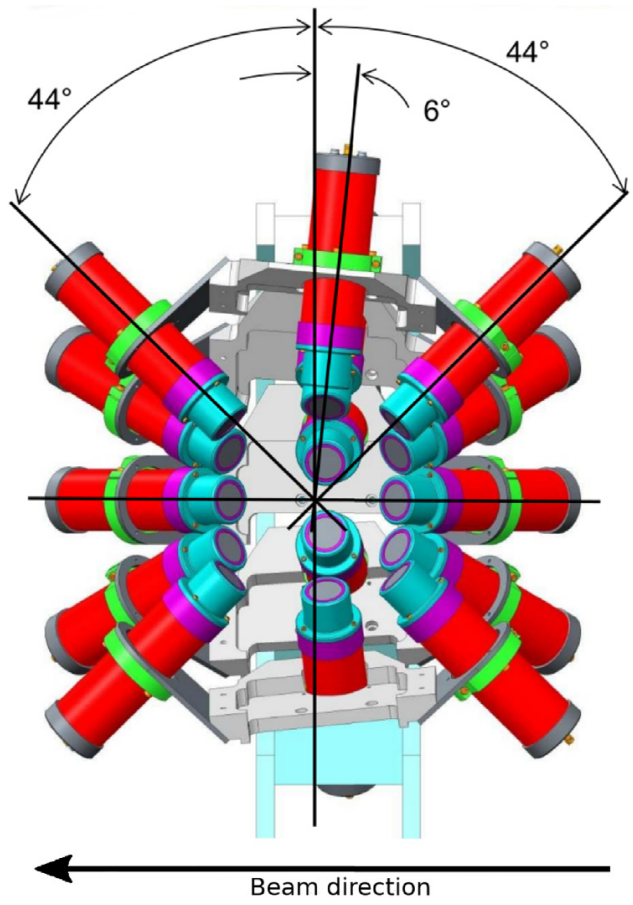

Fig. 4. (Colour online) Drawing of the FATIMA frame for DESPEC. The picture shows half of the structure with mounted detectors. The direction of travel of the incoming beam is indicated by the arrow at the bottom. From this view angle AIDA is situated on the right hand side.

Section 4. The technical design report [13] mentions future plans for extension of the setup. The design allows for two additional rings of smaller sized detectors at forward and backward angles.

In the standard configuration the detector faces have a distance of $16 \mathrm{~cm}$ from the focus point. This is also the closest distance that can be achieved for the fully equipped core FATIMA array. All measurements presented in this work done using the DESPEC frame at GSI were performed in the standard configuration. The ring design is rather flexible as all detectors can be freely moved along their cylinder axes. They can be extracted by a maximum of $7 \mathrm{~cm}$ from their standard position. This way the FATIMA array can accommodate larger or differently shaped implantation detector setups in the future, if this is desired. In principle, by drawing the detectors back, there is also space for additional detectors of smaller dimensions - for instance scintillator crystals equipped with space efficient silicon photomultipliers (SiPMs). Detectors like this could provide additional efficiency or active shielding.

\subsection{Electronics and data acquisition}

Peripheral electronics and a digital data acquisition system are part of the FATIMA setup. A list of the most important components is given in Table 1. Operation voltage for the photomultiplier tubes (PMTs) of the $\mathrm{LaBr}_{3}$ (Ce) detectors is supplied by a CAEN SY4527 unit equipped with three A1535D high voltage (HV) supply cards, housing 12 channels each. The typical stability of the cards is given as $<20 \mathrm{mV}$ by the manufacturer. Signal processing and data acquisition are based on VME electronics. The detector dynode signals are processed by V1751 digitisers. They are operated using the Digital Pulse Processing - Pulse Shape Discrimination (DPP-PSD) firmware provided by CAEN. The digitiser was chosen for its high sampling rate of $1 \mathrm{GS} / \mathrm{s}$. It is high enough to allow several samples on the signal rise time of the FATIMA $\mathrm{LaBr}_{3}(\mathrm{Ce})$ detector, which is about $6 \mathrm{~ns}$. This enables digital time pick off with sub-sample precision, for instance by a digital CFD algorithm (see, for instance, [22]). This is planned to be implemented in the future. Preliminary studies on this are reported in [23]. The energy 


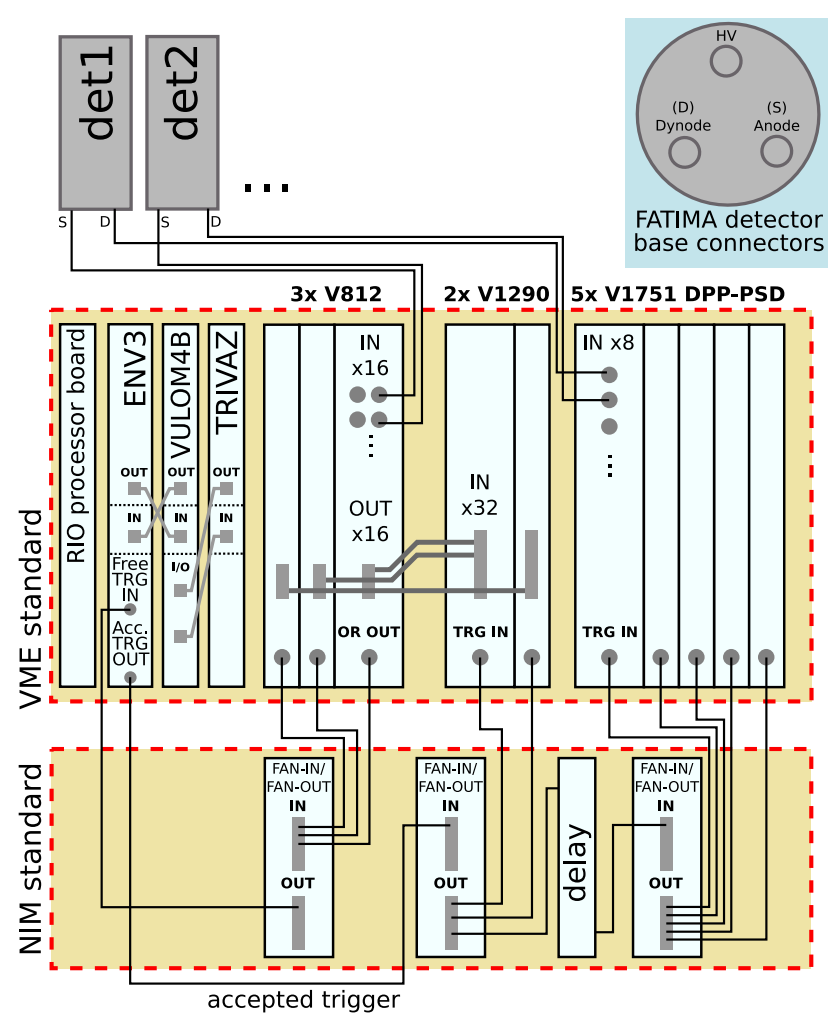

Fig. 5. (Colour online) Schematic depiction of the FATIMA data acquisition electronics. See text for details. The connectors at the base of the PMT and their labelling are shown in the top right.

Table 2

Overview of the main characteristics of the FATIMA detector array. The given values are for the fully equipped 36 detector core array, measured at GSI in the standard DESPEC configuration (distance of $16 \mathrm{~cm}$ from detector face to source position). Details are provided in Sections 3.1-3.3. Uncertainties are statistical uncertainties from the fit to the respective total spectrum. The remark " 60 Co coinc." in the last line means the $\gamma$-ray coincidence $1332 \mathrm{keV}-1173 \mathrm{keV}$ emitted from a ${ }^{60} \mathrm{Co}$ source was used.

\begin{tabular}{llll}
\hline Feature & Measurand & Value & Comment \\
\hline Energy resolution & FWHM $/ E_{\gamma}$ & $10.2(2) \%$ & at $122 \mathrm{keV}$ \\
& & $3.4(1) \%$ & at $779 \mathrm{keV}$ \\
& & $2.5(1) \%$ & at $1408 \mathrm{keV}$ \\
Full energy peak efficiency & & $11.6(4) \%$ & at $122 \mathrm{keV}$ \\
& & $3.6(1) \%$ & at $779 \mathrm{keV}$ \\
Coincidence resolving & FWHM & $2.1(1) \%$ & at $1408 \mathrm{keV}$ \\
& & $320(5) \mathrm{ps}$ & for ${ }^{60} \mathrm{Co} \mathrm{coinc.}$ \\
\hline
\end{tabular}

information is obtained via signal integration above a dynamically determined base line level (charge to digital conversion, QDC).

For time pick off of the anode signal of the detectors V812 constant fraction discriminators (CFDs) are used to minimise amplitude time walk. One module has 16 channels. The constant fraction delay can be set by jumpers on the boards to values between $4 \mathrm{~ns}$ and $20 \mathrm{~ns}$ in $4 \mathrm{~ns}$ steps. In the measurements presented in this work, the delay was set to $8 \mathrm{~ns}$. The constant fraction is fixed to $20 \%$ and is not adjustable. The module has no "time walk adjustment" setting to fine tune the zero-crossing for time pick off like it is, for example, featured on the Ortec 935 CFD [18]. Cross-talk has been observed between certain channels on the V812 which manifests itself as small side peaks in the time difference spectra involving these channels [24]. These channel combinations were avoided in the analysis of timing measurements.

For time measurements the CFD signals are fed into V1290 time to digital converters (TDCs). Each board has 32 channels accepting emitter-coupled logic (ECL) input signals. The time resolution is $25 \mathrm{ps}$ LSB (least significant bit) with a 21 bit range. This is not as precise as commonly used analogue time to amplitude converters (TACs), but is sufficient for sub-nanosecond timing. The $25 \mathrm{ps}$ time resolution is approximate. Measurements showed that this depends very much on the TDC and channel combination. From time difference spectra obtained from measurements with a high precision time calibrator we deduced a mean resolution of 24.6(5) ps in the least significant bit. The trigger, used as a time reference for incoming events, has a $25 \mathrm{~ns}$ clock cycle. The module has an optional multi-hit mode. The high channel density makes the system very space-efficient and transportable.

The VME modules are operated and read out using the GSI Multi Branch System (MBS) [25]. In full operation, FATIMA is part of the DESPEC data acquisition system, which is based on a common time stamp domain for triggers realised with White Rabbit [26]. The trigger scheme forsees a common dead time domain for FATIMA and a plastic detector which detects $\beta$-decay events of implanted ions.

Fig. 5 shows a schematic of the data acquisition electronics and signal connections for a FATIMA stand alone setup. In a common deadtime system with a fast plastic detector, the trigger will not directly derive from the CFDs, but will be a coincidence trigger. The condition in this case is at least one $\mathrm{LaBr}_{3}(\mathrm{Ce})$ detector and a valid plastic detector hit. Time correlation between FATIMA and the plastic detector can be achieved by sending a reference signal, in this case a trigger signal, to both systems for high precision time measurement (to the TDC V1290 in the FATIMA case). The accepted trigger output is not suited for this purpose due to its time precision which is on the scale of $5 \mathrm{~ns}$. Even with a high precision time reference an additional source of uncertainty is introduced into the system which results in a slightly worse time resolution - measurements showed this increase is on the order of 40 ps using two V1290 TDCs. The time reference is necessary if the time measurement is performed on separate modules whose time stamps cannot be synchronised. There are plans to unify the time measurement electronics of the FATIMA and $\beta$-detector systems to avoid having to use such a reference signal. A detailed description will be given in a later publication when tests of FATIMA-plastic timing have been concluded.

Outside of GSI, the software MIDAS [27], developed at STFC Daresbury Lab, UK, was used for stand-alone operation and when part of FATIMA was integrated with another detector system. Two examples of measurements where MIDAS was used are presented in Section 4. A V1495 logic module is part of the system. It can be used to control and program the readout and trigger logic. See Section 4.1 for an example of an application of this module.

\section{Characteristics}

In this section the main characteristics of the FATIMA detector system are described. All source measurements were performed at the S4 station at GSI during the autumn of 2018. Table 2 gives an overview of the most important results for the core array as it existed in 2018. Characteristics of the individual detectors are given in Table 3 towards the end of this section.

\subsection{Energy calibration and energy resolution}

The energy response of scintillator detectors is in general not linear over a wide dynamic range. In the case of the FATIMA detectors the degree of non-linear response is sensitive to the voltage at which the PMT is operated. In most cases a second degree polynomial is sufficient for an energy calibration with a precision of about $0.2 \mathrm{keV}$ up to $1.5 \mathrm{MeV}$; however in some cases, and for large absolute voltages, higher degrees are necessary. Fig. 6 demonstrates the non-linearity for one detector operated at $-1100 \mathrm{~V}$. The plot shows the residual of a fit of peak position in QDC channels to the corresponding energies from a ${ }^{152} \mathrm{Eu}$ source for the range from $244 \mathrm{keV}$ to $1408 \mathrm{keV}$ using three different polynomial functions. The linear fit does not describe the data well while a polynomial of second degree (square) yields a significant 
Table 3

List of all FATIMA detectors. The individual crystal and PMT numbers are given for all detectors. For characterisation each detector was operated at a voltage so that the output signal of the $1332 \mathrm{keV}$ line from a ${ }^{60} \mathrm{Co}$ source had an amplitude of $-600 \mathrm{mV}$. The typical voltage to achieve this was $-1050 \mathrm{~V}$. The individual time resolution is given in coincidence with detector number 1 . The average relative energy resolution at $779 \mathrm{keV}$ is $3.4(2) \%$ with standard deviation given as uncertainty. The average FWHM of the prompt coincidence time distributions obtained with the two ${ }^{60} \mathrm{Co}$ lines is $320 \mathrm{ps}$ with 5 ps standard deviation in this data set. *) determined in separate measurement with similar conditions.

\begin{tabular}{|c|c|c|c|c|}
\hline $\begin{array}{l}\text { Detector } \\
\text { number }\end{array}$ & $\begin{array}{l}\text { Crystal } \\
\text { ID }\end{array}$ & $\begin{array}{l}\text { PMT } \\
\text { ID }\end{array}$ & $\begin{array}{l}\text { Energy resolution } \\
\text { (\%@779 keV) }\end{array}$ & $\begin{array}{l}\text { Time resolution } \\
\text { FWHM (ps) }\end{array}$ \\
\hline 1 & A3835 & FA1339 & $3.30(5)$ & ref. \\
\hline 2 & A3828 & FA1631 & $3.40(5)$ & 303(9) \\
\hline 3 & A3834 & FA1418 & $3.40(5)$ & $320(10)$ \\
\hline 4 & A3830 & FA0816 & $3.30(5)$ & $305(9)$ \\
\hline 5 & A3837 & FA0800 & $3.40(5)$ & $312(9)$ \\
\hline 6 & A3829 & FA1578 & $3.30(5)$ & $324(10)$ \\
\hline 7 & A3836 & FA1071 & $3.50(5)$ & $320(10)$ \\
\hline 8 & A3832 & FA1623 & $3.30(5)$ & 292(9) \\
\hline 9 & A3815 & FA1171 & $3.50(5)$ & $335(10)$ \\
\hline 10 & A3833 & FA1075 & $3.30(5)$ & 298(9) \\
\hline 11 & A3838 & FA0613 & $3.30(5)$ & $452(15)$ \\
\hline 12 & A3839 & FA0634 & $3.30(5)$ & 292(9) \\
\hline 13 & A3814 & FA0643 & $3.30(5)$ & 289(8) \\
\hline 14 & A3817 & FA0657 & $3.50(5)$ & $337(10)$ \\
\hline 15 & A3808 & FA0412 & $3.30(5)$ & $303(9)$ \\
\hline 16 & A3812 & FA0600 & $3.50(5)$ & $303(9)$ \\
\hline 17 & A3811 & FA1614 & $3.20(5)$ & $316(9)$ \\
\hline 18 & A3809 & FA1736 & $3.40(5)$ & $318(10)$ \\
\hline 19 & A3810 & FA1415 & $3.50(5)$ & $320(10)$ \\
\hline 20 & A3816 & FA1162 & $3.30(5)$ & $326(10)$ \\
\hline 21 & A3821 & FA1627 & $3.40(5)$ & $308(9)$ \\
\hline 22 & A3813 & FA1122 & $3.30(5)$ & 287 (9) \\
\hline 23 & A3819 & FA0687 & $3.30(5)$ & $323(10)$ \\
\hline 24 & A3826 & FA1613 & $3.50(5)$ & $322(10)$ \\
\hline 25 & A3824 & FA1165 & $3.40(5)$ & $330(10)$ \\
\hline 26 & A3822 & FA0846 & $3.60(5)$ & $309(9)$ \\
\hline 27 & A3823 & FA1831 & $3.30(5)$ & 303(9) \\
\hline 28 & A3831 & FA0829 & $3.20(5)$ & $306(9)$ \\
\hline 29 & A3825 & FA1158 & $4.00(6)$ & $403(12)$ \\
\hline 30 & A3820 & FA1735 & $3.50(5)$ & 299(9) \\
\hline 31 & A3818 & FA8202 & $3.30(5)$ & $322(10)$ \\
\hline 32 & A8292 & FA2091 & $3.40(5)$ & $308(9)$ \\
\hline 33 & A8293 & FA2092 & $3.40(5)$ & $314(9)$ \\
\hline 34 & A8294 & FA2096 & $3.80(6)$ & $343(10)$ \\
\hline 35 & A8295 & FA2097 & $3.30(5)^{*}$ & $336(10)^{*}$ \\
\hline 36 & A8296 & FA2145 & $3.10(4)^{*}$ & $321(10)^{*}$ \\
\hline
\end{tabular}

improvement. Only a small additional improvement is achieved by using a polynomial function of third degree (cube). The average energy calibration precision found during the measurements was $\pm 0.2 \mathrm{keV}$ for the fit with a third degree polynomial.

It should be noted that the gain of PMTs is sensitive to large sudden changes in count rate (more precisely changes of the signal output current), which should be kept in mind when preparing calibration measurements. Furthermore, gain drifts and shifts are a well known issue for detectors with PMTs (e.g. [28]). See Section 4.1 for a description of gain drifts in FATIMA during a long measurement with a ${ }^{252} \mathrm{Cf}$ source. These drifts are usually corrected through a gainmatching procedure during the first stage of analysis. This is especially important for measurements which take several weeks under the same conditions. If the detectors are properly warmed up, i.e. set to operation voltage to stabilise several hours before the measurement, the drift is usually small over the course of several days, leading to a degradation of energy resolution of several percent. However, changes in experiment parameters such as the ambient temperature, count rate, or HV instabilities can lead to more dramatic effects. Count rate effects are especially prevalent during in-beam measurement when the beam current is unstable or if sometimes there is no beam for many hours. Long term irreversible effects on the gain are called fatigue in the literature [29]. For FATIMA these have not yet been studied.

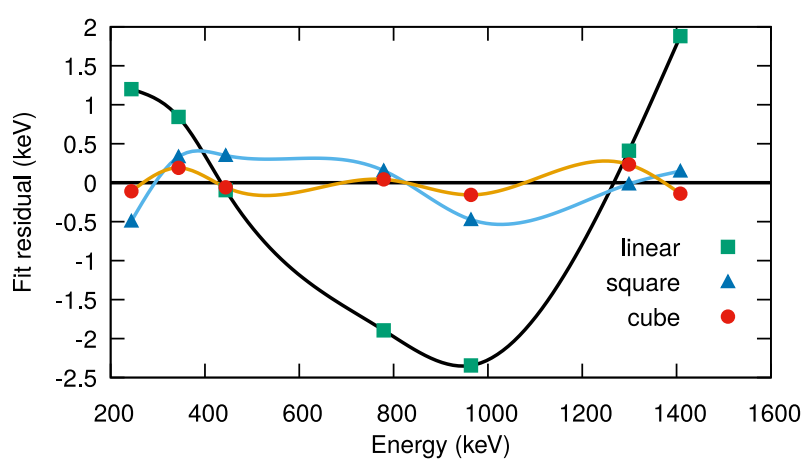

Fig. 6. (Colour online) Fit residuals of energy calibrations using polynomial functions of different degree for one FATIMA detector operated at $-1100 \mathrm{~V}$. The points are connected with splines to guide the eye. See text for details.
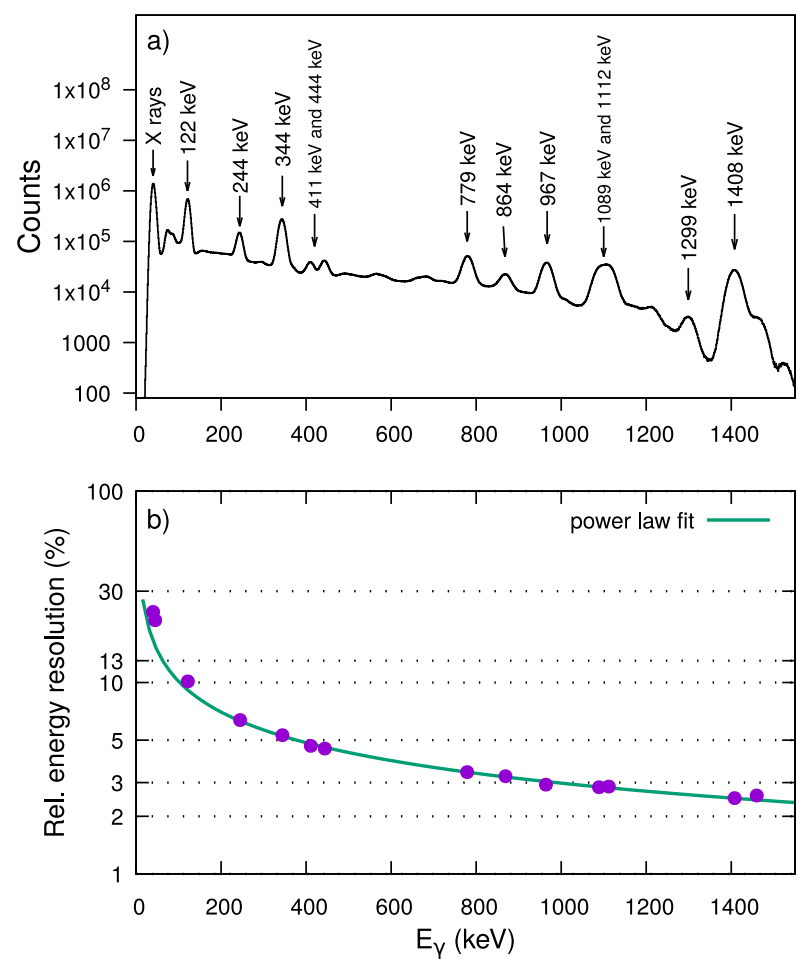

Fig. 7. (Colour online) (a) Gamma-ray energy spectrum of a ${ }^{152} \mathrm{Eu}$ source measured using FATIMA equipped with 34 detectors (added single spectra). (b) Relative energy resolution FWHM/ $\mathrm{E}_{\gamma}$ obtained for the spectrum shown in (a). A power law was fitted to the data above $200 \mathrm{keV}$. The exponent found in the fit is $-0.53(1)$.

The energy resolution of detectors which use $\mathrm{LaBr}_{3}(\mathrm{Ce})$ as scintillation material is among the best of this type of detectors. The reason for this is the extremely high light yield achieved with this material [30]. Fig. 7(a) shows the sum of the individual calibrated energy spectra of $34 \mathrm{LaBr}_{3}(\mathrm{Ce})$ detectors ${ }^{1}$ mounted on the FATIMA DESPEC frame at the S4 station at GSI. A ${ }^{152} \mathrm{Eu}$ source was placed at the centre of the array. The PMTs were operated at a voltage so that the $1332 \mathrm{keV}$ line of a ${ }^{60} \mathrm{Co}$ source results in an output signal of $-600 \mathrm{mV}$. The average voltage was around $-1050 \mathrm{~V}$. It can be seen that the doublet of $411 \mathrm{keV}$ and $444 \mathrm{keV}$ is well resolved while the doublet at $1089 \mathrm{keV}$ and $1112 \mathrm{keV}$ is not. This is typical for $\operatorname{LaBr}_{3}(\mathrm{Ce})$ detectors of this size (cf. e.g. [19,31]). Fig. 7(b) shows a plot of the total relative energy resolution of the

1 Two of the 36 FATIMA $\mathrm{LaBr}_{3}(\mathrm{Ce})$ detectors were continuously used as reference detectors for tests with the DESPEC fast plastic detector during the time these test measurements were taken. 


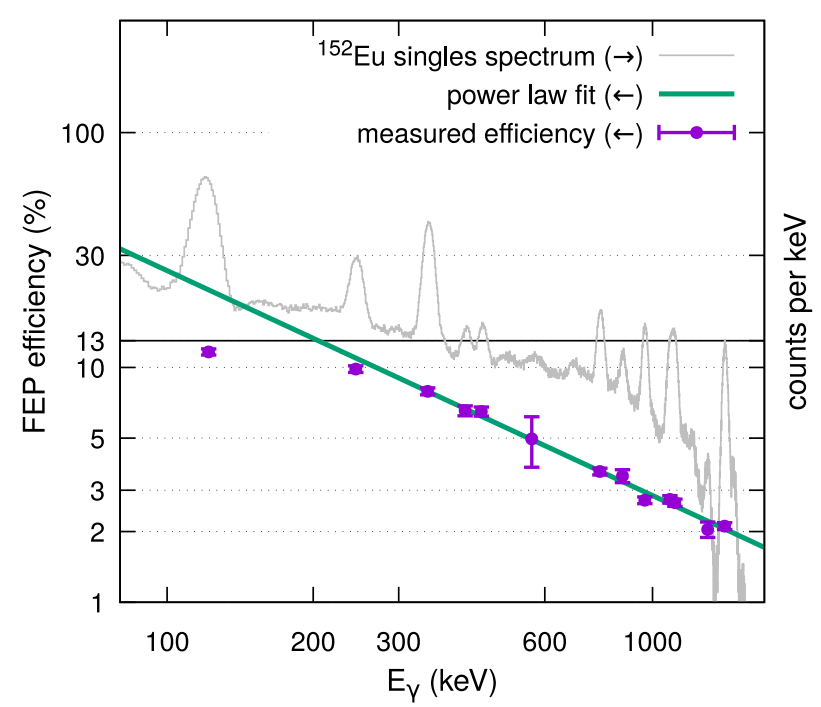

Fig. 8. (Colour online) Absolute full energy singles peak efficiency as a function of energy, measured with a ${ }^{152}$ Eu source and the FATIMA array at S4, GSI, equipped with 34 detectors. The result was scaled to represent the efficiency for a fully equipped array (36 detectors). The black horizontal line indicates the part of the solid angle covered by the 36 detector faces. Data points above $300 \mathrm{keV}$ were fitted with a power law. This yielded an exponent of $-0.96(2)$.

array for the range up to $1400 \mathrm{keV}$. It is evident that the resolution follows a power law for energies above $100 \mathrm{keV}$. The resolution of each individual detector found in the measurement discussed above is given in Table 3.

\subsection{Full energy peak efficiency}

The full energy peak efficiency of a $\gamma$-ray detector array is an important measure for rate estimates during the planning phase of an experiment. The absolute full energy peak efficiency of FATIMA in the DESPEC frame, with the nominal distance of $160 \mathrm{~mm}$ from the face of the crystal to the focus point is shown as a function of energy in Fig. 8. From the fit with a power law for values above $300 \mathrm{keV}$ we obtain a total full energy peak efficiency of $2.9(1) \%$ at $1 \mathrm{MeV}$ for the array. Single detector efficiencies are shown in Fig. 9. The values were obtained from a measurement with a ${ }^{152} \mathrm{Eu}$ source positioned in the focus point on a source tray made of the thermoplastic polymethyl methacrylate (PMMA). The tray itself was only a few millimetres thick, but $10 \mathrm{~cm}$ wide. This led to material being present between the source and certain detectors looking at the source from a small angle from below. The attenuation of low energy $\gamma$ rays for these detector positions (pos. 4, 9, 15, 21, 28, and 33) can be seen clearly in Fig. 9.

Note that the efficiency values quoted here should not be used directly for rate estimates, but should rather be used for cross checks of simulations with a setup closer to that of the particular experiment. The presence of AIDA detectors or additional plastic detectors for $\beta$ detection will reduce the absolute full energy peak efficiency of FATIMA - especially at $\gamma$-ray energies below $500 \mathrm{keV}$. Additional attenuation is caused by the AIDA snout, a metal case which shields the sensitive segmented detectors.

The addition of the extra rings with smaller detectors will make it possible to enhance the geometrical efficiency by approaching the implantation point.

\subsection{Timing characteristics}

The timing characteristics of DESPEC FATIMA are given here only in the context of $\gamma-\gamma$ delayed coincidence measurements using FATIMA in standalone mode as tests with the $\beta$ detectors designed for upcoming DESPEC experiments at GSI have not yet been concluded.

The two main characteristics presented are the time-resolution and the time walk of the complete system for $\gamma-\gamma$ fast timing measurements.

Time resolution in this context is the full width at half maximum (FWHM) of a prompt time difference distribution. This value is energy dependent (more precisely amplitude dependent) in both the start and the stop branch of the timing circuit. The time resolution of the full array is obtained from the sum of time-difference spectra of all combinations of FATIMA. For this purpose the time spectra are aligned by applying a constant shift to each detector time which is obtained from the coincidence with a reference detector (in this case detector 1). The minimum shift is the minimum bin value of the fine time stamp delivered by the V1290 TDC, which is $25 \mathrm{ps}$. The position of a time distribution measured with this TDC can be measured with a precision greater than $25 \mathrm{ps}$, however, the shift always has to be a multiple of this least significant bit. This can cause slight differences between the average time resolution - obtained from the average of the coincidence time resolutions of the individual combinations - and the time resolution of the combined time spectrum obtained by the summing of the aligned time spectra.

For the measurement of the timing characteristics the PMT voltage of each detector was adjusted so that the $1332 \mathrm{keV}$ transition produced a pulse height of $-600 \mathrm{mV}$. This way the input to the CFD is as similar as possible for all detectors which ensures a similar timing behaviour as well. The individual coincidence time resolutions using detector 1 gated on the $1332 \mathrm{keV}$ transition as a reference are given in Table 3 . The average CTR is 320 ps FWHM. On closer inspection it can be seen that the resolution is notably poorer for the combination 1-11 and 129. This is due to faulty CFD channels. From here on combinations involving these detectors have been excluded.

For actual lifetime measurements the sum of all combinations is used. It has been demonstrated by Régis et al. [31] that this is a valid approach for centroid shift measurements with large fast timing arrays. Fig. 10(a) shows the combined time spectrum from a ${ }^{60} \mathrm{Co}$ measurement with 32 FATIMA detectors. The resolution here is 334.3(4) ps FWHM, which is slightly worse than the average resolution of $320 \mathrm{ps}$. The shape of the prompt response is symmetric and can be reproduced very well with a gaussian distribution. A measurement of the lifetime of the first excited $2^{+}$state at $122 \mathrm{keV}$ in ${ }^{152} \mathrm{Sm}$ is shown in Fig. 10(b).

The time walk, or residual time walk, that still persists after the signal has been discriminated in the CFD, is a very important characteristic for the application of the centroid shift method. Applying this method, lifetimes down to less than 10 ps can be measured by $\gamma$ $\gamma$ delayed coincidence using the FATIMA array [32]. For this purpose the prompt response curve (PRC) of the system, i.e. the position of the centroid of a prompt time distribution as function of energy in the start and stop branch, has to be measured using full energy peaks. The PRC is determined by the energy dependent residual time walk of both the start, and the stop branch of the timing system. It can be measured via coincident $\gamma$ ray cascades emitted by a ${ }^{152} \mathrm{Eu}$ source to a precision of a couple of ps over a broad energy range from about $200 \mathrm{keV}$ to about $1400 \mathrm{keV}$ [33]. To achieve this high accuracy also below $200 \mathrm{keV}$ and above $1400 \mathrm{keV}$, other $\gamma$ ray coincidence sources have to be used. See [34] for a recent publication on fast-timing in the low energy region.

The PRC of DESPEC FATIMA in the current configuration for a reference energy of $344 \mathrm{keV}$ is shown in Fig. 11. From the fit residual we can estimate the uncertainty of the prompt response calibration to be \pm 5 ps for $\gamma$-ray energies above $200 \mathrm{keV}$ and below $1400 \mathrm{keV}$. The curve shown in Fig. 11 is similar to the one obtained from a measurement performed at Argonne National Laboratory in which the same detectors and electronics were used [32]. 


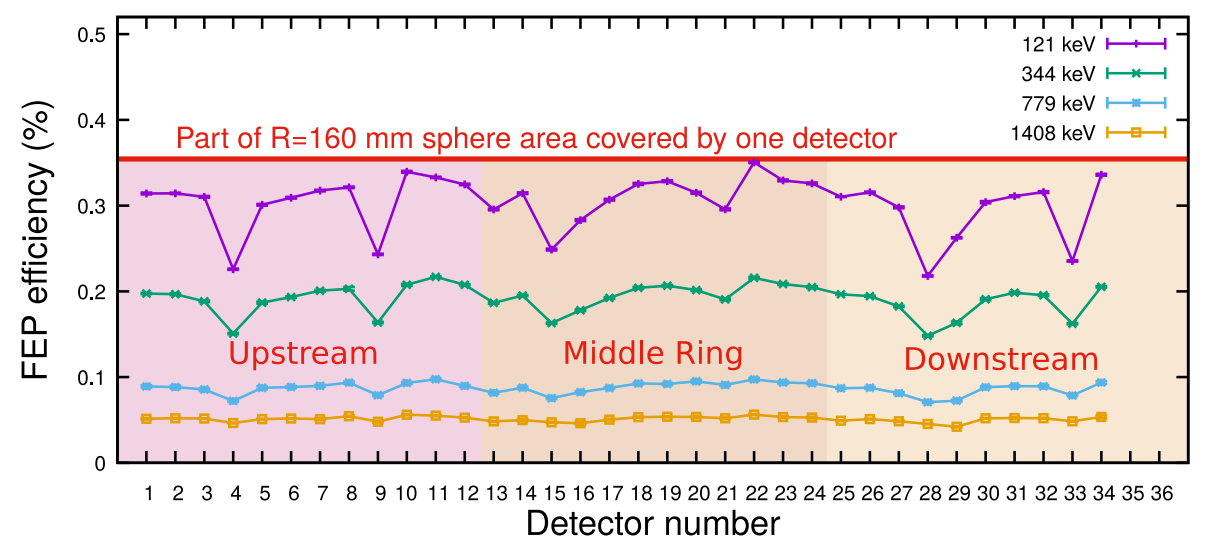

Fig. 9. (Colour online) Single detector efficiency from the same measurement as shown in Fig. 8. See text for details.
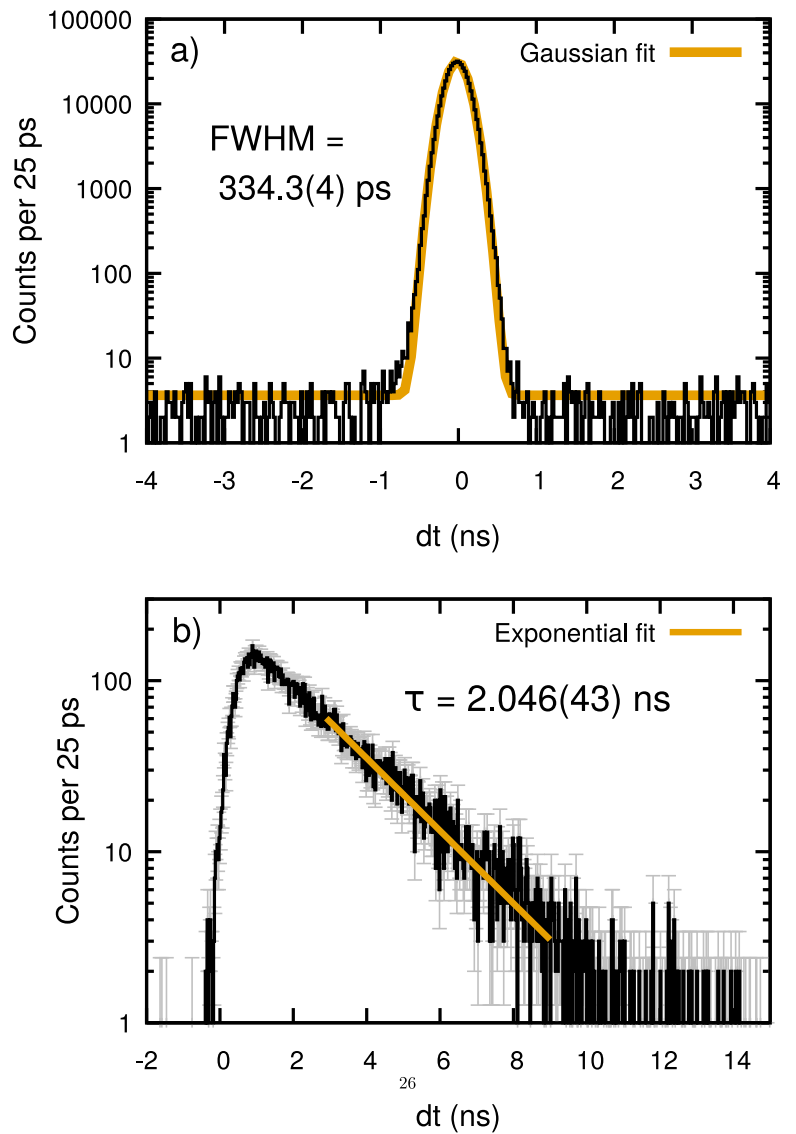

Fig. 10. (Colour online) Time spectra measured with FATIMA equipped with 34 detectors. (a) Time distribution from the prompt ${ }^{60} \mathrm{Co}$ decay $\gamma$ cascade. (b) Delayed coincidence time distribution of the $122 \mathrm{keV}-1408 \mathrm{keV}$ cascade in ${ }^{152} \mathrm{Sm}\left({ }^{152} \mathrm{Eu}\right.$ source). The mean lifetime $\tau$ is measured to be 2.046(43) ns using an exponential fit to the slope. This is in good agreement with the literature value of $\tau=2.020(20)$ ns [35].

\subsection{Time correction for extended implantation area}

The implantation area of the AIDA detector is expected to have a FWHM of several centimetres. The good position resolution of AIDA will help to identify implantations and link them to delayed $\beta$ decay events. However, this extended beam profile also leads to a possible variation of the path lengths between the point of emission and detection in individual FATIMA detectors on the order of centimetres. This time of flight (ToF) difference can actually be resolved by the $\mathrm{LaBr}_{3}(\mathrm{Ce})$
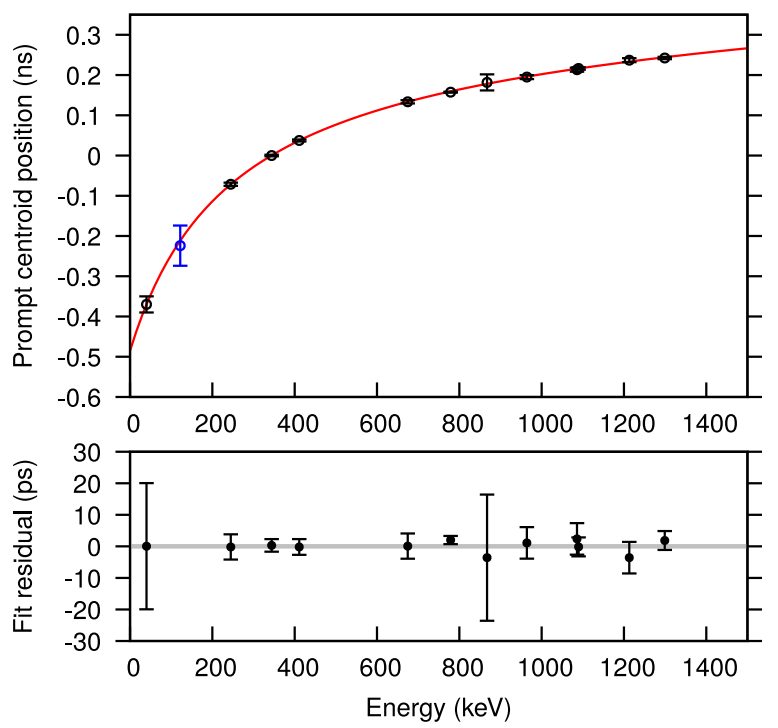

Fig. 11. Prompt response curve (PRC) for the FATIMA array measured using coincident $\gamma$ transitions emitted by a ${ }^{152} \mathrm{Eu}$ source for a reference energy of $344 \mathrm{keV}$. The centroid obtained for the $1408 \mathrm{keV}-122 \mathrm{keV}$ coincidence from the spectrum in Fig. 10 (b) is drawn in blue, corrected by the lifetime of the $2^{+}$state $i^{152} \mathrm{Sm}$ given in [35]. The bottom panel shows the fit residual. See text for more details. (For interpretation of the references to colour in this figure legend, the reader is referred to the web version of this article.)

detectors. Possible AIDA configurations have a sensitive implantation area of $8 \times 8 \mathrm{~cm}^{2}$ or $24 \times 8 \mathrm{~cm}^{2}$, with a matching FRS beam profile.

As the point of implantation or $\beta$-decay during an experiment is known via the AIDA array, an event-by-event correction can be implemented using the known distances and angles. Several tests were performed with sources positioned at different points away from the focus point in order to study the effect on the time spectrum and also to verify the correction procedure. A recent study on this performed at the RITU focal plane at the University of Jyväskylä using eight FATIMA detectors can be found in [36].

Fig. 12 shows time spectra from the tests performed at GSI. First the usual corrections for the constant relative time shifts between the detectors were obtained from a measurement with a ${ }^{60} \mathrm{Co}$ source at the centre of the array, giving the average time resolution of the array, which is around $320 \mathrm{ps}$. Then the source was positioned on the source table at a position $32 \mathrm{~mm}$ above the centre. By applying only the correction factors for the centre position, the time distribution is noticeably wider due to the different travel times of the $\gamma$ rays for different detector combinations. With the correction procedure applied, based on the known detector positions and the position of the source, the 


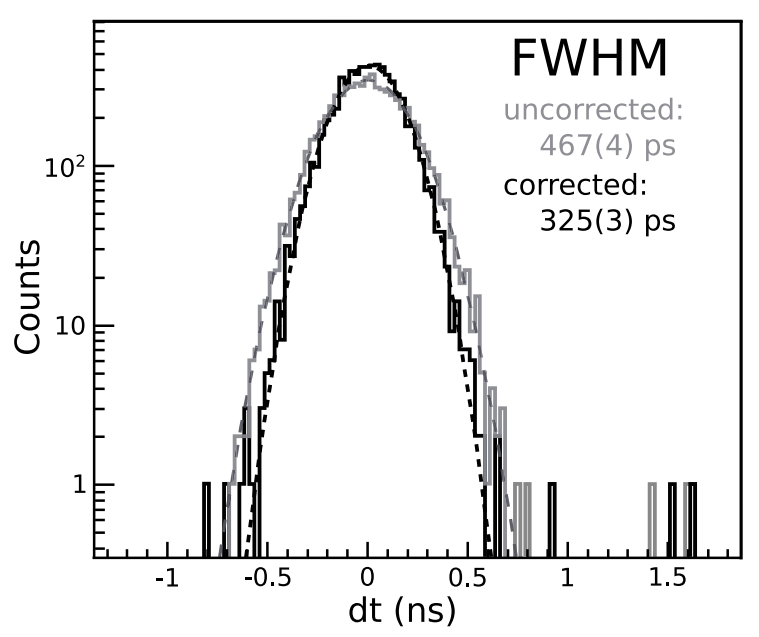

Fig. 12. Time difference spectrum taken with FATIMA and a ${ }^{60} \mathrm{Co}$ source $32 \mathrm{~mm}$ above the focus position. The different flight paths of the $\gamma$ rays for the different detector combinations leads to a deteriorated time resolution (red/grey). After correction (blue/black) the average resolution of $\sim 320 \mathrm{ps}$ is retained. (For interpretation of the references to colour in this figure legend, the reader is referred to the web version of this article.)

average time resolution is retained, which shows that the implemented algorithm works.

\subsection{Background and scattering between detectors}

As mentioned in Section 2.1, due to radioactive constituents of the $\operatorname{LaBr}_{3}(\mathrm{Ce})$ scintillator material, a significant background rate due to intrinsic activity is always present in measurements with this type of detector. Fig. 13(a) shows an energy spectrum of the background measured with one FATIMA detector at the S4 station at GSI (all 36 core detectors were mounted, and equipped with lead shields). The background spectrum is clearly dominated by the internal activity due to the decay of ${ }^{138} \mathrm{La}$ and the decay chain products of ${ }^{227} \mathrm{Ac}$ ( $\alpha$ lines around $2 \mathrm{MeV}$ ). Ac is a chemical homologue to La and remains the material after chemical purification. The strongest line is the $1438 \mathrm{keV}$ $2^{+} \rightarrow 0^{+} \gamma$ emitted after electron capture decay to ${ }^{138} \mathrm{Ba}$ (often detected as a sum peak together with an X-ray). Electrons from $\beta^{-}$decay to ${ }^{138} \mathrm{Ce}$ are also visible, along with the $789 \mathrm{keV} 2^{+} \rightarrow 0^{+} \gamma$ line from that decay. The latter is visible as the energy sum of electron and $\gamma$. The ${ }^{40} \mathrm{~K}$ decay line at $1460 \mathrm{keV}\left(2^{+} \rightarrow 0^{+}\right.$in $\left.{ }^{40} \mathrm{Ar}\right)$ is also visible. Fig. 13(b) shows the total background rate for individual detectors, including X-rays from the ${ }^{138} \mathrm{La}$ decay, that were observed during this measurement. The average background rate (internal and external) per detector is 73 counts per second, which is the expected magnitude given a typical activity of $\mathrm{LaBr}_{3}(\mathrm{Ce})$ material of $1 \mathrm{~Bq} / \mathrm{cm}^{2}$ [20].

The rates for decay experiments with exotic nuclei is typically on the same level as the background and therefore online single spectra in these measurements can be expected to resemble the one in Fig. 13(a). The ${ }^{138} \mathrm{La}$ decay only produces one $\gamma$ ray with energy $1438 \mathrm{keV}$ which is only in coincidence with the corresponding $\mathrm{K} \mathrm{X}$-ray at approximately $32 \mathrm{keV}$. As this background is not correlated with any $\beta$ decay from implanted ions it becomes negligible in $\gamma-\gamma$ or $\beta-\gamma$ coincidence measurements.

One of the main sources of background in fast timing measurements with scintillator detectors is $\gamma$ rays which are Compton scattered. Either they are scattered in the detector itself, with the outgoing $\gamma$ ray escaping the scintillator, or they scatter in a neighbouring detector or in the surrounding material and the scattered $\gamma$ ray is then detected. The problem this type of background is that it is time correlated with the physical reaction that produced the $\gamma$ ray, but it cannot be attributed to any particular transition based on its energy as the distribution of the
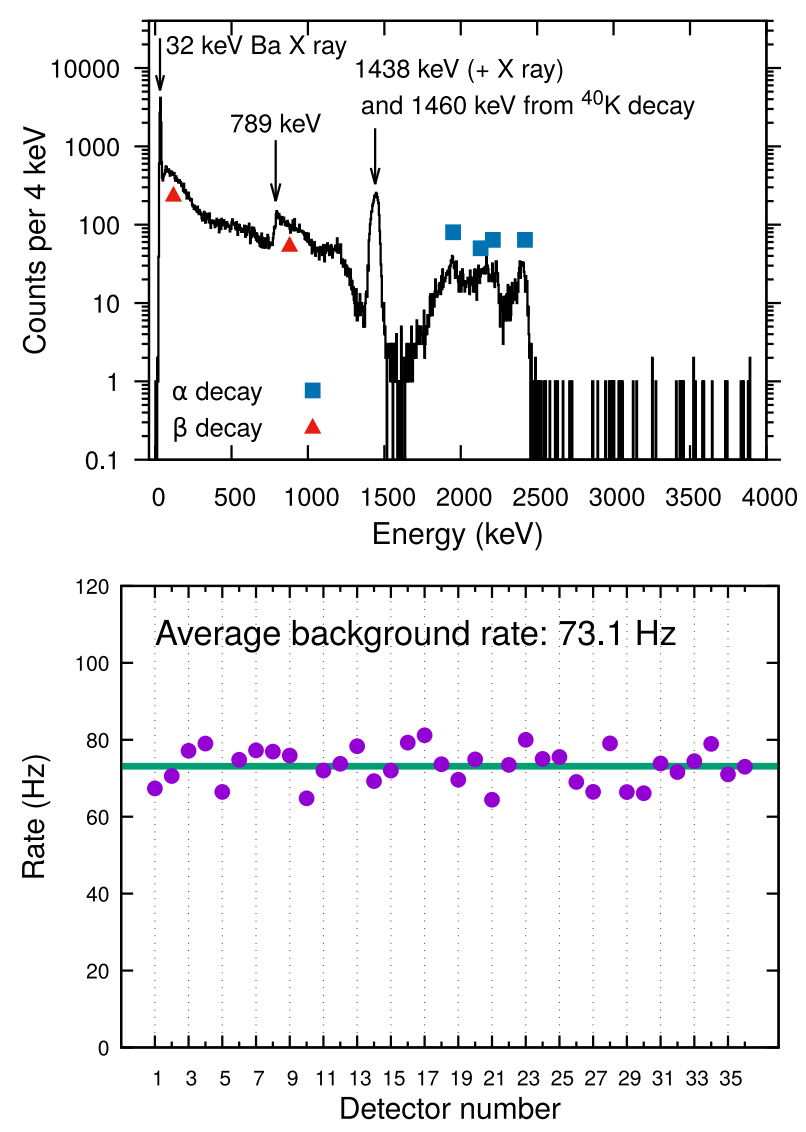

Fig. 13. (a) Background energy spectrum of FATIMA detector 20 mounted at $S 4$ at GSI. See text for details. (b) Background rates for individual FATIMA detectors. With 34 FATIMA detectors installed the total trigger rate during this measurement was $2.6 \mathrm{kHz}$.

Compton scattered $\gamma$ rays is continuous. Due to the facts mentioned above, the time behaviour of these scattered $\gamma$ rays is different to that of those originating directly from the source.

Due to the close geometry of the three rings, the major external source of Compton continuum events in any individual FATIMA detector are the detectors surrounding it. Specially shaped lead shields of $5 \mathrm{~mm}$ thickness, which fit around the crystal cap of the detectors, can suppress most of the low energy scattered $\gamma$ rays.

In some cases it can be advantageous to not use the lead shielding. The crystals surrounding a particular detector can work as that detector's active Compton suppression. Assists in the improvement of the peak-to-background ratio of the whole spectrometer as a certain part of Compton scattered events can actually be identified and discarded by vetoing coincidences between neighbouring detectors in the analysis. This is not possible if a large part of the scattered $\gamma$ rays are stopped in the lead. Of course this way real full energy coincidences between neighbours are also suppressed. In principle add-back can be applied as well, to recover part of the Compton scattered $\gamma$ rays for the full energy peak. However, this was not tested yet under real experimental circumstances with the FATIMA array.

Removing the lead shields can be a disadvantage in experiments with comparably high count rates where it might be desired to apply a trigger condition of more than one detected $\gamma$ ray in FATIMA. Removing the lead shields will drastically increase the number of events with two or more detected $\gamma$ rays. This leads to an increase of the free trigger rate without increasing the selectivity, which is potentially not desired.

\section{FATIMA on the road}

The 36 detectors of the FATIMA core system, as well as the electronics, have been used for measurements at various research facilities 


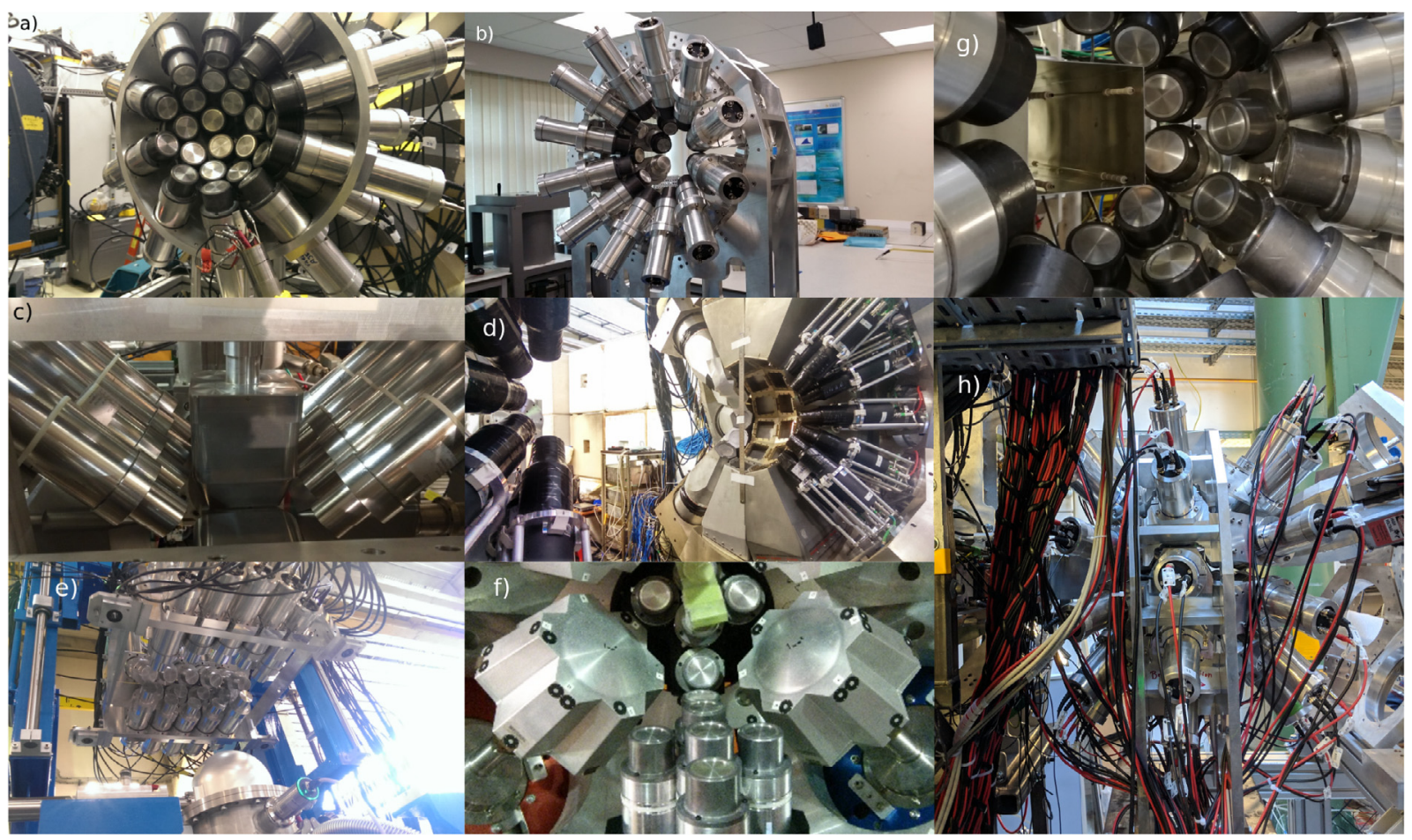

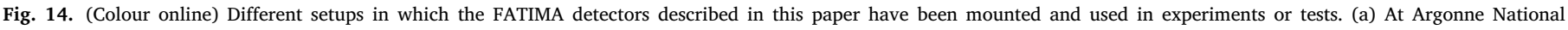

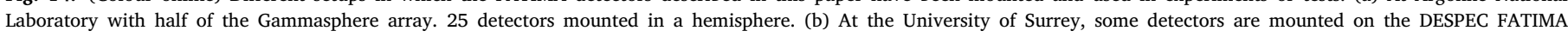

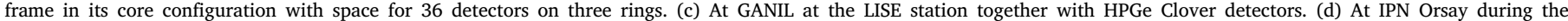

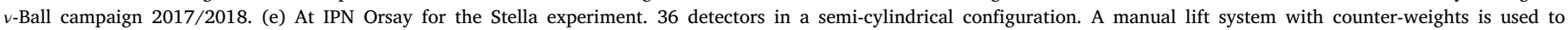

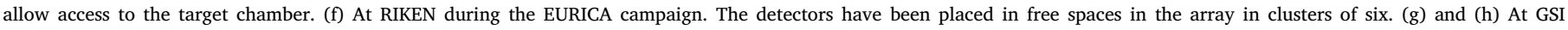
in the S4 cave in the configuration designed for use in the DESPEC station for FAIR (AIDA snout and rack to the left, beam from left to right).

before their first installation at GSI. As each different site and experiment has different demands concerning space constraints, distance, and number of detectors, and therefore different solutions had to be found for each of these applications. Fig. 14 shows the holding structures that have been built for different experiments. The FATIMA detectors described in this paper were used, for example, at Argonne National Laboratory with Gammasphere [32] (see Section 4.1), at GANIL with AGATA and VAMOS [37], at the IPN Orsay with Stella [38] (see Section 4.2) and in the beginning of the v-Ball campaign [39-41], and at RIKEN during the EURICA campaign $[42,43]$. In all these cases the detectors were fixed in place using custom designed clamps. When combined with VAMOS, additional shielding had to be applied due to fringing magnetic fields interfering with the photomultiplier tubes of the detectors (see Section 4.3). Detectors discussed in this work have also been in use during the EXILL+FATIMA campaign at the ILL, Grenoble, France (see for instance [44]), and at measurements with the RoSPHERE array in Bucharest, Romania (see e.g. [45]).

\subsection{At Argonne with digital Gammasphere}

The first experiment in which a large set of FATIMA detectors was used together with the VME electronics and MIDAS based data acquisition system, was with Digital Gammasphere at Argonne National Laboratory, USA $[46,47]$. Twenty five FATIMA detectors were arranged in a hemisphere (photo in Fig. 14(a)) while half of Gammasphere (51 High-Purity Germanium (HPGe) detectors) filled the other hemisphere. A ${ }^{252} \mathrm{Cf}$ spontaneous fission source was placed at the centre. The source measurement took place in winter 2015 and lasted for several weeks into January 2016 - a time where usually no beam is delivered to Gammasphere. The aim was to perform HPGe-gated $\gamma-\gamma \operatorname{LaBr}_{3}(\mathrm{Ce})$ fast timing measurements in the produced fission products. A detailed description of the setup and conditions during the experiment can be found in [32]. Results from this measurement are presented in [48-52].
Integration of the FATIMA DAQ with Digital Gammasphere was achieved via a MyRIAD module [53] which was installed in the FATIMA VME crate. A clock on the module was synchronised with the master clock of the Gammasphere DAQ. The two systems collected data independently. Later the data were merged based on the common time stamp. For a more detailed description the reader is referred to [32] and references therein.

The event-by-event data read-out was controlled by a global FATIMA trigger. The trigger logic was implemented via a V1495 VME module. The logic on the FPGA was programmed at Daresbury Laboratory and the module and programme are quite flexible and can also be used to integrate FATIMA with other systems. For instance with the Narval system which is used at GANIL and other nuclear physics laboratories in France. (For this purpose a specially designed CENTRUM mezzanine card can be mounted in the bottom one of the three slots available on the V1495.) The VME crate was controlled through a MIDAS system running on a PC workstation. Communication was facilitated through an A3818 PCI card on the PC end, and a V2718 crate controller in the VME crate. This allows for readout and operation of the VME modules via VME bus through the backplane. It was found that each read or write operation takes several $\mu \mathrm{s}$. All together, including data readout of four V1751 digitisers, this led to a total read-out time of typically $400 \mu$ s per event. The trigger logic does not accept a new trigger until read-out of the current event is complete in order to unambiguously correlate the QDC and TDC measurements to a single event. This means that the read-out time establishes a hard limit on the accepted trigger rate of about $2.5-3 \mathrm{kHz}$. Based on the average background rate of $71 \mathrm{~Hz}$ per detector (see Section 3.5), the background rate for 25 FATIMA detectors is on the order of $1.7 \mathrm{kHz}$. Single FATIMA detector rates during the experiment were on the scale of $3 \mathrm{kHz}$. For this reason a coincidence trigger of $2 \mathrm{LaBr}_{3}(\mathrm{Ce})$ detectors within $200 \mathrm{~ns}$ and at least one additional Gammasphere detector within $500 \mathrm{~ns}$ was set up, which lowered the free trigger rate during the measurement while selecting interesting events. 


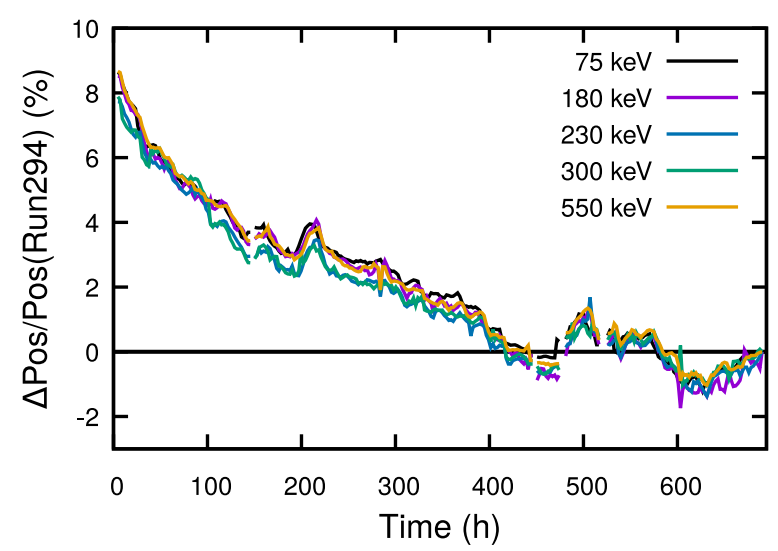

Fig. 15. (Colour online) Gain drift of one FATIMA detector during the measurement with a ${ }^{252} \mathrm{Cf}$ source at Argonne. The position of five peaks is tracked, relative to a reference run 294 at the end of the measurement. $\Delta \mathrm{Pos}=\operatorname{Pos}(\mathrm{t})-\operatorname{Pos}($ Run294). Gaps in the data are due to runs that were excluded because of problems with the data acquisition.

Recent developments might make a separate time measurement redundant. Progress in digital time pick off of fast scintillator signals using digitisers with relatively low sampling rates (e.g. a $500 \mathrm{MHz}$ digitiser was used during the $v$-Ball campaign [32]) have made the application of this technique more and more practical (and affordable) for the purpose of fast timing with scintillators. In principle the CAEN V1751, with its $1 \mathrm{GHz}$ sampling rate, is able to do this as well. The time difference spectrum prompt resolution we observe by using the digital CFD algorithm provided by the CAEN firmware is comparable with analogue TACs, but the actual measurement resolution we found to be around $64 \mathrm{ps}$ in the least significant bit. This is a very strong challenge to perform picosecond range lifetime measurements using the centroid shift analysis.

The measurement with the ${ }^{252} \mathrm{Cf}$ source lasted several weeks, during which the setup was in constant, uninterrupted operation. HV supply was stable and the rate of $\gamma$ rays (and neutrons) incident on the detectors was to very good approximation constant during the measurement. For this reason the experiment is ideal to demonstrate the long term stability of the PMT used in the FATIMA detectors. As mentioned in Section 3.1 there are several effects that influence the gain of the PMT apart from the applied voltage. The long term drift, which is caused by wear mainly of the last dynode due to constant bombardement with electrons, is called the fatigue of the PMT [29]. Fig. 15 shows the gain change of one detector by tracking the position of several prominent peaks in the energy spectrum. A steady trend to lower gain can be seen with small higher frequency changes superimposed. Gain change is generally small over the range of a couple of days. However, some larger jumps can be identified which seem to happen randomly.

This drift has to be corrected for during the analysis. In our case this was done by gain matching the single detectors to the last runs of the campaign. A technical description of the gain matching procedure can be found in [24].

Some publications with results from this campaign were provided at the beginning of this section. As an example, the lifetime measurement of the first excited $4^{+}$state in ${ }^{100} \mathrm{Zr}$ is included; a $\mathrm{LaBr}_{3}(\mathrm{Ce})$ double coincidence projection energy spectrum with a Gammasphere gate on the $2^{+} \rightarrow 0^{+}$transition in ${ }^{100} \mathrm{Zr}$ is shown in Fig. 16(a). The coincident transitions from further up the ground state band can be seen clearly. As common in prompt fission experiments, transitions from the fission partners - in this case ${ }^{148} \mathrm{Ce}-$ also appear in coincidence. Fig. 16(b) shows the time-difference spectra obtained by gating on the $4^{+} \rightarrow 2^{+}$ and $6^{+} \rightarrow 4^{+}$transitions in the $\mathrm{LaBr}_{3}(\mathrm{Ce})$ detectors. In this case the centroid difference method [31], a technique based on the centroid shift method, was used to extract the lifetime from these time distributions.
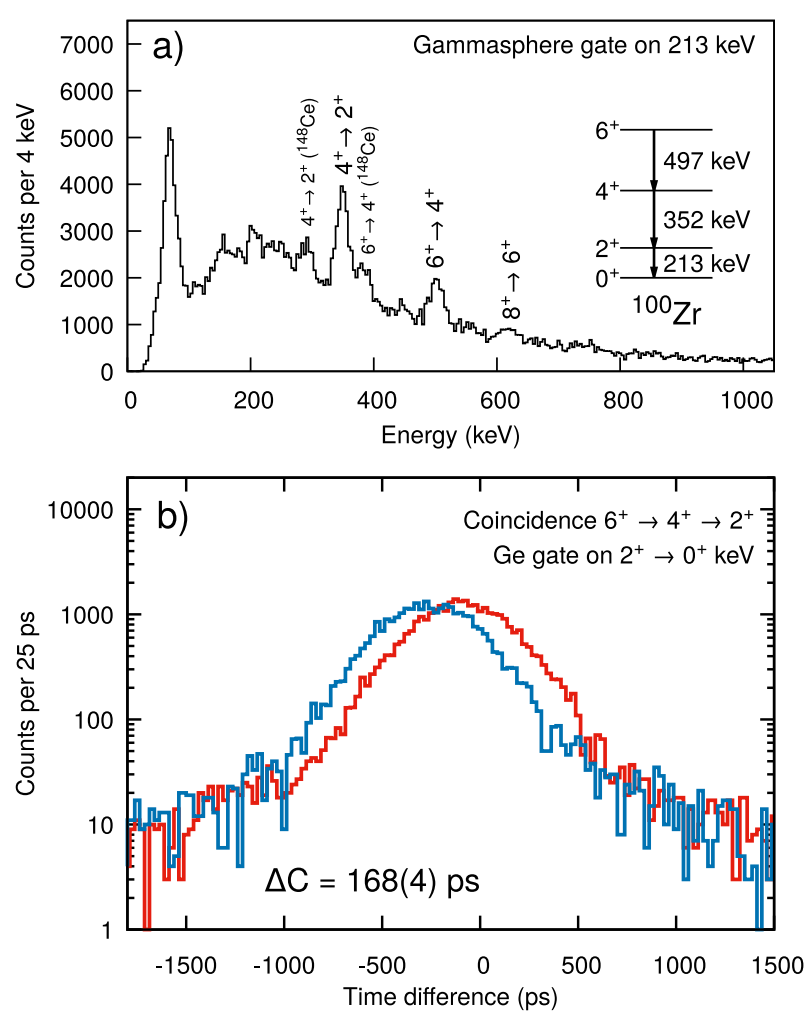

Fig. 16. (a) Projection of a gated $\mathrm{LaBr}_{3}$ (Ce) $\gamma-\gamma$ coincidence energy matrix. An energy gate condition on the $2^{+} \rightarrow 0^{+}$transition in ${ }^{100} \mathrm{Zr}$ is set on Gammasphere, background is subtracted. This matrix was used to set the gates for the lifetime measurement of the $4^{+}$ state. (b) Delayed (timing start gate on $6^{+} \rightarrow 4^{+}$transition; red/black) and anti-delayed (timing stop gate on $6^{+} \rightarrow 4^{+}$; blue/grey) $\mathrm{LaBr}_{3}(\mathrm{Ce})$ time difference distributions used to determine the lifetime of the $4^{+}$state in ${ }^{100} \mathrm{Zr}$. The final result of $\tau_{4^{+}}=34(11) \mathrm{ps}$ is obtained from the measured centroid difference after correction for residual time walk and background contributions. (For interpretation of the references to colour in this figure legend, the reader is referred to the web version of this article.)

The background was treated as described in [48]. The FWHM of the distributions is approximately $650 \mathrm{ps}$. The final result of $\tau_{4^{+}}=34(11) \mathrm{ps}$ is in agreement with a recent $\gamma \gamma$ timing measurement done during the EXILL FATIMA campaign which yielded $\tau_{4^{+}}=37(4)$ [54]. It is within two sigma of a differential plunger measurement performed in 2002 by Smith et al. [55] which yielded 53.4(5) ps. This example demonstrates the capabilities of FATIMA to measure lifetimes in the region of tens of picoseconds, even in situations with high background contributions. A closer description of the analysis procedure for this lifetime can be found in [24].

\subsection{At IPN Orsay with Stella}

A fruitful collaboration in which FATIMA is involved is the Stella experiment at the IPN Orsay, France. Here the FATIMA detectors are used to detect $\gamma$ rays emitted after $\left({ }^{12} \mathrm{C},{ }^{12} \mathrm{C}\right)$ sub-barrier fusion, working in coincidence with the Stella particle detectors. A description of the setup and first results can be found in references [56,57], and [58].

For this experiment sub-nanosecond timing was not crucial. The nanosecond time stamp provided by the CAEN V1751 digitiser was sufficient to obtain clean coincidences with the particle detectors employed. Therefore a free running data acquisition system was implemented for FATIMA. Synchronisation with the particle detector data stream was achieved via the built-in phase-locked loop capability of the V1751 digitiser. The particle detector acquisition clock was used as an external clock which was then distributed to the five modules. Stability was very good - clock drifts over 24 -h measurements never 


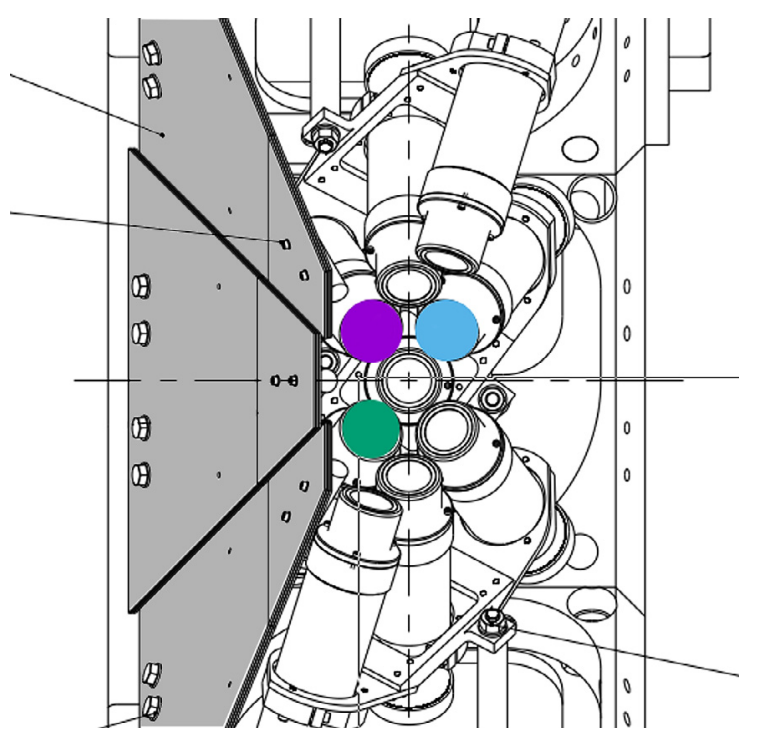

Fig. 17. This figure shows the positions on the EXOGAM frame in front of VAMOS which were mounted with detectors during the test measurement. The view is parallel to the ground and orthogonal to the beam axis. The VAMOS quadrupole magnet is situated on the left. The detector mounted at the position coloured in purple was a $\mathrm{CeBr}_{3}$ detector which was equipped with an individual magnetic shield can. The $\mu$-metal shield plates on the left are shown shaded grey. (For interpretation of the references to colour in this figure legend, the reader is referred to the web version of this article.)

exceeded 2 ticks ( 1 ns ticks on the V1751, 8 ns ticks for the particle DAQ). Time alignment was checked by feeding a signal into both data acquisition systems simultaneously in certain intervals on the order of minutes. These were then used to verify time alignment online and during the offline analysis. By starting all the cards simultaneously using an external NIM pulse, the digitiser time stamp offsets were small, which made analysis much easier by avoiding the determination of large time offsets which can be in the millisecond range if the boards are started one after the other through software.

MIDAS was used for control and acquisition of the FATIMA system. Data were recorded on a dedicated PC and then streamed to the central workstation where some simple checks were performed on data rate and time alignment with the particle DAQ. For coincidence analysis data files of the two systems were merged into a single data tree based on their time stamp.

Due to the very low rates during this experiment the single spectra of each $\mathrm{LaBr}_{3}(\mathrm{Ce})$ detector are governed by its intrinsic activity. This feature can be used to perform gain matching based on the known shape of the background. An algorithm for this was developed by M. Heine for this experiment [57]. This application can be very interesting for DESPEC experiments as well, as the rates there are also expected to be only slightly above the background level.

\subsection{Influence of magnetic fields at GANIL with the VAMOS spectrometer}

The PMT of the FATIMA detectors come with magnetic shielding appropriate to ensure operation in the geomagnetic field, which has an average strength of the order of $\approx 10 \mu \mathrm{T}$. When mounted on the EXOGAM frame, just before VAMOS [59] (see Fig. 17), the fringing fields created by the VAMOS quadrupole magnet can reach several $10 \mathrm{mT}$ at certain detector positions. Magnetic fields of this strength noticeably affect the electron trajectories in the PMT. This leads to a worsened energy and time resolution and eventually, for large enough fields, renders the detector unusable. The field strength in the location of the EXOGAM frame has a strong dependence on the angle around the beam axis and on the distance to the VAMOS quadrupole magnet. It was found to be strongest on the ring closest to VAMOS and on the diagonal positions close to angles of $45^{\circ}$ with respect to the horizontal plane. The field in these positions was measured using a Hall probe in absence of any shields. We observed maximum absolute values of $15 \mathrm{mT}$ at $200 \mathrm{~A}$ and $54 \mathrm{mT}$ at $600 \mathrm{~A}$ with an estimated uncertainty of $0.5 \mathrm{mT}$. Common setting for VAMOS during experiments use currents between these two values.

In order to counter the effect of the fringing field, additional shielding has to be incorporated. $\mu$-metal plates were installed between VAMOS and the $\operatorname{LaBr}_{3}(\mathrm{Ce})$ detectors. These shields were made up of 4 layers of $2 \mathrm{~mm}$ thick plates of $\mu$-metal (see Fig. 17). Tests have been performed to investigate the effect of this shielding. All tests have been done with the detectors operated at $-1200 \mathrm{~V}$. Due to the fact that most detectors were used at the Stella setup in Orsay during the time of the tests, we used two $\mathrm{LaBr}_{3}(\mathrm{Ce})$ detectors and a $\mathrm{CeBr}_{3}$ detector from the National Physical Laboratory, UK, for the tests. These detectors had the same PMT model as the FATIMA detectors. $\mathrm{CeBr}_{3}$ crystals perform only slightly worse than $\mathrm{LaBr}_{3}(\mathrm{Ce})$ concerning energy and time resolution, and have no internal activity [60]. Results from these tests are shown in Fig. 18. It can be seen that in a position with the strongest fringing fields, energy resolution as well as gain are stable and within acceptable limits up to a VAMOS current of about $300 \mathrm{~A}$. For higher currents resolution and gain quickly deteriorate. On positions farther away from the magnet the detectors operate in acceptable limits up to a VAMOS current of $600 \mathrm{~A}$. For common settings used to separate medium-heavy ions VAMOS currents of $400 \mathrm{~A}$ and more are necessary. The effect of the plates was therefore deemed to be insufficient and additional shielding was considered. This consisted of individual shielding cans with a thickness of $2 \mathrm{~mm}$, which cover the crystal and PMT of the detector. As can be seen from Fig. 18(left) this shielding, in combination with the plates, is effective for VAMOS currents up to $600 \mathrm{~A}$ on the positions with the strongest fringing fields.

In order to test the effects of the magnetic fields on the time resolution and the time walk of the detectors, measurements were performed using a ${ }^{60} \mathrm{Co}$ source. One detector was gated on the $1332 \mathrm{keV}$ transition. The other detector was then gated on different energies in the Compton continuum of the $1173 \mathrm{keV}$ transition to measure the energy dependence of FWHM and centroid position of the prompt time distribution. Results of these measurements are shown in Fig. 19. As only one shield was available as a prototype for this test measurement, this detector, marked purple in Fig. 17 (det1), was chosen as a reference. From the analysis of the gain behaviour it is clear that the detector marked blue in Fig. 17 (det2) is hardly affected by the fringing field, while the detector marked green (det3) is strongly affected. The grey data points were taken with a VAMOS current of $0 \mathrm{~A}$ and serve as a reference for normal behaviour of the PMT. The measurement taken at $150 \mathrm{~A}$ shows no significant change with det 3 gated on $1332 \mathrm{keV}$ and det 1 on the Compton continuum (black data points). At $400 \mathrm{~A}$, time resolution as well as time walk are strongly affected for det3 gated on $1332 \mathrm{keV}$ and det1 gate on the Compton continuum as well as for the opposite case (red data points). This shows clearly that the EXOGAM $\mu$-metal shield plates alone are not sufficient to enable proper functioning of the detectors with these VAMOS currents. The effect is less pronounced if the gate on det 3 is fixed on $1332 \mathrm{keV}$ (red circles) than if it is gated on the Compton continuum (red squares). This is because the effect of the distortion is larger for lower energies, a sign that the shielded detector is less affected in the energy region of the Compton continuum. For the detector combination det1-det2, also at a VAMOS current of $400 \mathrm{~A}$, there is no significant effect on time resolution or centroid position (blue data points). This shows that the additional shielding is sufficient to guarantee proper timing behaviour for typical experimental conditions during VAMOS measurements.

Note that during actual measurements with VAMOS additional magnetic field sources would be present. These are typically two strong permanent magnets which are positioned on the top and bottom of the part of the target chamber flanged to VAMOS. These magnets are placed in order to deflect delta electrons produced by the ion beam. 

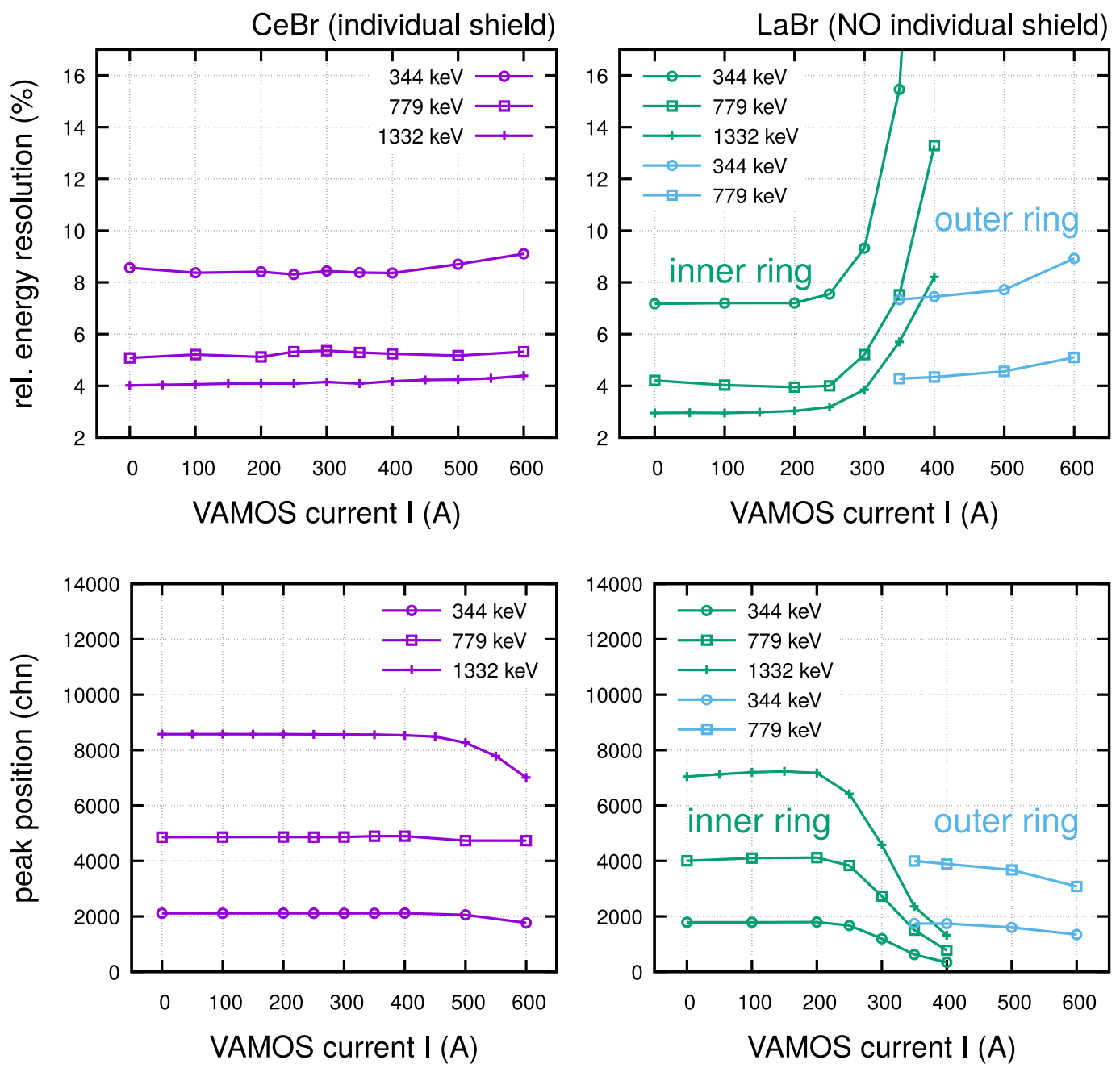

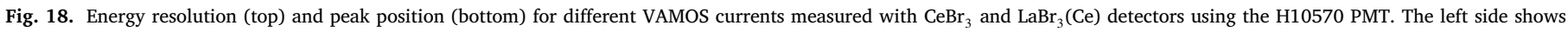

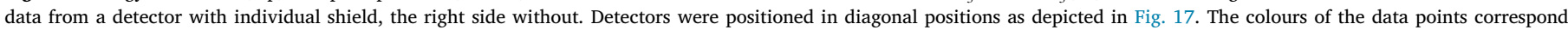

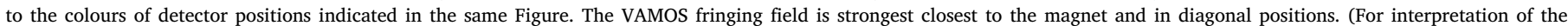
references to colour in this figure legend, the reader is referred to the web version of this article.)

The strength of these magnets was not quantitatively measured, but it appeared from the spectra that for the detectors closest to the magnets the field was comparable to that produced by the quadrupole fringing fields during the experiment. The effect of these additional magnets is not reflected in the measured data presented here.

In summary, the measurements indicate, that the shielding of the FATIMA PMTs with $\mu$-metal plates between the detectors and VAMOS and the additional individual shielding cans provide enough protection to ensure normal operation of the detectors up to at VAMOS current of at least $500 \mathrm{~A}$. The sensitivity of the PMTs to magnetic fields is a well known draw-back of this type of detector, which was already anticipated in the FATIMA TDR. Other types of photomultipliers are less sensitive, like Si-photomultipliers. Their application with $\mathrm{LaBr}_{3}(\mathrm{Ce})$ as scintillator material for fast-timing measurements is currently studied by several groups of the collaboration.

\section{Summary and outlook}

The basic characteristics and capabilities of the DESPEC FATIMA core system have been presented. The performance is in accordance with the technical design report submitted by the FATIMA collaboration [13]. Energy and time resolution are as expected for the type of detector and crystal size. The system is suitable to measure lifetimes via $\gamma-\gamma$ delayed coincidence from the region of tens of picoseconds into the nanosecond range. An algorithm for correction of flight-path differences that occur for an extended source, like the FRS beam spot, has been implemented and tested. The test measurements have not raised any points that could cause problems in the combination of the array with a fast $\beta$ detector to apply the $\beta-\gamma$ fast timing method, which is the main purpose of FATIMA at the decay spectroscopy station planned for FAIR.

The setup as well as the electronics components that are currently used for the system have been described. The three ring configuration has been set up and tested with sources at GSI. The FATIMA infrastructure at GSI is ready for upcoming measurements. The integration of the current system with other detector systems, like Digital Gammasphere and Stella, have briefly been described and an overview of other applications has been given. A test measurement at GANIL showed the influence of external magnetic fields on the behaviour of the FATIMA detectors.

For the future, a change of the timing electronics, either a different CFD-TDC combination or a completely digital system, as they have become available recently, has the potential to improve the time resolution and walk characteristics. An all digital solution could also improve the dead time issue that comes with an unbuffered system like the one currently used, and make application of the array for experiments with larger detection rates feasible. 

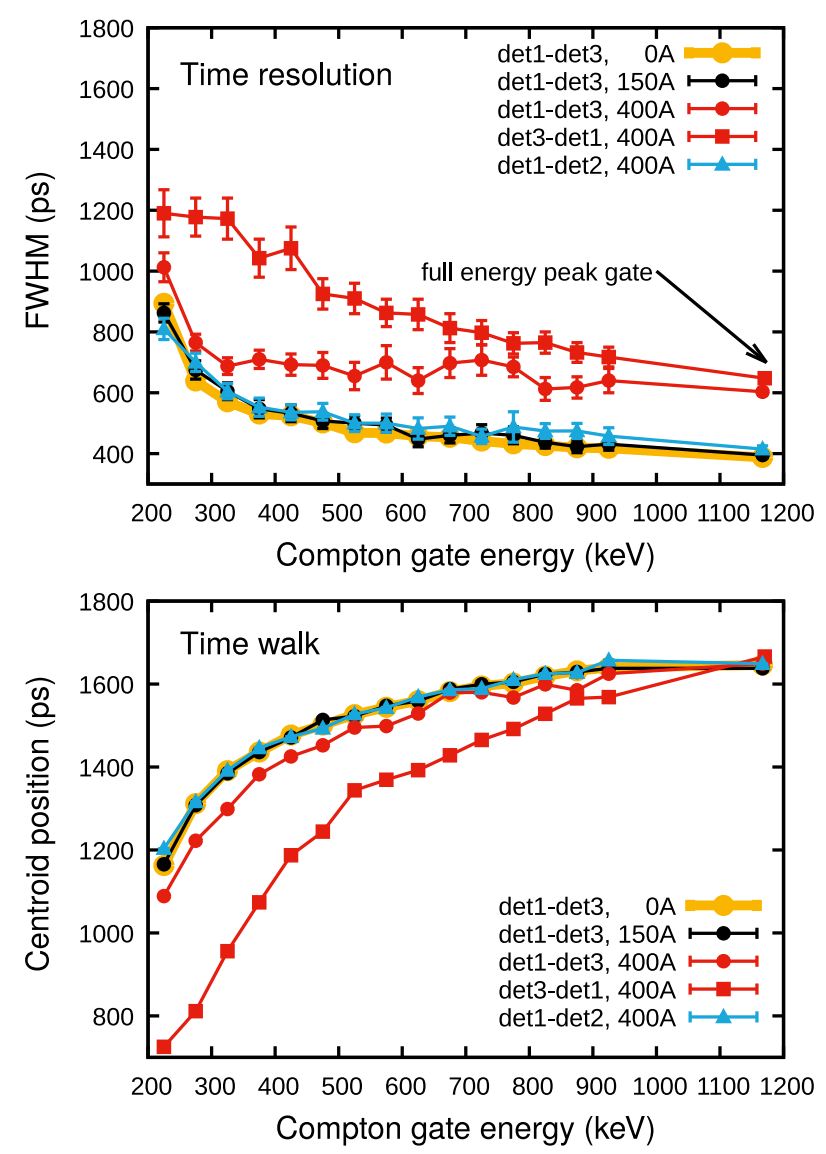

Fig. 19. Effect of VAMOS fringing fields on time resolution (top) and prompt centroid position (bottom) of detectors using the H10570 PMT. In the description, the convention for $\operatorname{det} \mathrm{X}$-detY is that $\operatorname{det} \mathrm{X}$ is gated on the $1173 \mathrm{keV}$ Compton continuum, detY on the $1332 \mathrm{keV}$ full energy peak. In the bottom picture the black, blue and yellow lines lie almost exactly on top of each other. See text for more details. (For interpretation of the references to colour in this figure legend, the reader is referred to the web version of this article.)

\section{Declaration of competing interest}

The authors declare that they have no known competing financial interests or personal relationships that could have appeared to influence the work reported in this paper.

\section{CRediT authorship contribution statement}

M. Rudigier: Writing - original draft, Investigation, Formal analysis. Zs. Podolyák: Writing - review \& editing, Funding acquisition, Conceptualization. P.H. Regan: Writing - review \& editing, Funding acquisition, Conceptualization. A.M. Bruce: Writing - review \& editing, Funding acquisition, Conceptualization. S. Lalkovski: Investigation, Validation. R.L. Canavan: Investigation, Formal analysis, Software. E.R. Gamba: Investigation, Formal analysis, Software. O. Roberts: Software, Conceptualization, Investigation. I. Burrows: Resources. D.M. Cullen: Conceptualization, Validation. L.M. Fraile: Writing - review \& editing, Funding acquisition, Conceptualization. L. Gerhard: Investigation . J. Gerl: Conceptualization, Supervision, Project administration. M. Gorska: Conceptualization, Supervision, Project administration. A. Grant: Resources. J. Jolie: Conceptualization, Funding acquisition . V. Karayonchev: Investigation. N. Kurz: Software, Data curation. W. Korten: Conceptualization, Investigation, Resources. I.H. Lazarus: Software, Data curation. C.R. Nita: Investigation, Formal analysis. V.F.E. Pucknell: Software, Data curation. J.-M. Régis: Validation, Methodology. H. Schaffner: Software, Data curation. J. Simpson: Conceptualization, Funding acquisition. P. Singh: Investigation, Formal analysis.
C.M. Townsley: Project administration. J.F. Smith: Conceptualization, Validation. J. Vesic: Investigation, Formal analysis.

\section{Acknowledgements}

The core FATIMA array was funded by the UK Science and Technology Facilities Council (UK STFC) under the grants ST/G000751/1, ST/G000697/1, ST/G000638/1, ST/G000689/1, ST/G000727/1, and ST/P006698/1.

This work is partially supported by the UK STFC under grant numbers ST/L005743/1 and ST/P005314/1. P.H. Regan acknowledges support from the UK Department of Business, Energy and Industrial Strategy (BEIS) via the National Measurement System (NMS). R.L. Canavan acknowledges support from the STFC UK Nuclear Data Network and the Marion Redfern Trust of the University of Surrey. This work was partly funded by the BMBF, Germany under grants 05P15PKFNA and 05P19PKFNA, and by the Spanish MINECO through projects FPA201565035-P, RTI2018-098868-B-I00 and the NuPNET network FATIMA (PRI-PIMNUP-2011-1338).

\section{References}

[1] B. Rubio, Decay spectroscopy (DESPEC) at the new Fair-Nustar facility, Internat. J. Modern Phys. E 15 (2006) 1979.

[2] Z. Podolyák, From RISING to HISPEC/DESPEC, Nucl. Instrum. Methods Phys. Res. 266 (2008) 4589.

[3] P.H. Regan, From RISING to the DESPEC fast-timing project within NUSTAR at FAIR: Sub-nanosecond nuclear timing spectroscopy with $\mathrm{LaBr}_{3}$ scintillators, Appl. Radiat. Isot. 70 (2012) 1125.

[4] B. Rubio, T. Nilsson, NuSTAR, Nucl. Phys. News 16 (2006) 9-14.

[5] S. Lalkovski, Z. Podolyák, M. Bentley, A.M. Bruce, I. Lazarus, R. Lemmon, P.H. Regana, J. Simpson, The UK NuStAR project, Acta Phys. Polon. B 47 (2016) 637.

[6] R. Krücken, The NuStAR facility at FAIR, J. Phys. G 31 (2005) S1807.

[7] H. Geissel, H. Weick, M. Winkler, G. Münzenberg, V. Chichkine, M. Yavor, T. Aumann, K.H. Behr, M. Böhmer, A. Brünle, K. Burkard, J. Benlliure, D. CortinaGil, L. Chulkov, A. Dael, J.-E. Ducret, H. Emling, B. Franczak, J. Friese, B. Gastineau, J. Gerl, R. Gernhäuser, M. Hellström, B. Jonson, J. Kojouharova, R. Kulessa, B. Kindler, N. Kurz, B. Lommel, W. Mittig, G. Moritz, C. Mühle, J. Nolen, G. Nyman, P. Roussell-Chomaz, C. Scheidenberger, K.-H. Schmidt, G. Schrieder, B.M. Sherrill, H. Simon, K. Sümmerer, N.A. Tahir, V. Vysotsky, H. Wollnik, A.F. Zeller, The Super-FRS project at GSI, Nucl. Instrum. Methods Phys. Res. B 204 (2003) 71-85.

[8] C.J. Griffin, T. Davinson, A. Estrade, D. Braga, I. Burrows, P.J. Coleman-Smith, T. Grahn, A. Grant, L.J. Harkness-Brennan, G. Kiss, M. Kogimtzis, I.H. Lazarus, S.C. Letts, Z. Liu, G. Lorusso, K. Matsui, S. Nishimura, R.D. Page, M. Prydderch, V.H. Phong, V.F.E. Pucknell, S. Rinta-Antila, O.J. Roberts, D.A. Seddon, J. Simpson, S.L. Thomas, P.J. Woods, $\beta$-Decay studies of r-process nuclei using the advanced implantation detector array (AIDA), JPS Conf. Proc. 14 (2017) 020622.

[9] T. Davinson, et al., Technical Report for the Design, Construction and Commissioning of the Advanced Implantation Detector Array (AIDA), FAIR Publications, 2008.

[10] Z. Podolyák, Studies of exotic nuclei with advanced radiation detectors, Radiat. Phys. Chem. 95 (2014) 14.

[11] B. Longfellow, P.C. Bender, J. Belarge, A. Gade, D. Weisshaar, Commissioning of the $\mathrm{LaBr}_{3}(\mathrm{Ce})$ detector array at the National Superconducting Cyclotron Laboratory, Nucl. Instrum. Methods Phys. Res. A 916 (2019) 141-147.

[12] B. Olaizola, Griffin Collaboration, GRIFFIN's fast-timing array, APS Meeting Abstracts, APS Division of Nuclear Physics Meeting Abstracts 2016 (2016) PD.004.

[13] L.M. Fraile, et al., Technical Design Report for the DESPEC Fast Timing Array, FAIR Publications, 2015.

[14] H. Mach, R.L. Gill, M. Moszynski, A method for picosecond lifetime measurements for neutron-rich nuclei, (1) outline of the method, Nucl. Instrum. Methods Phys. Res. A 280 (1989) 49.

[15] L.M. Fraile, Fast-timing spectroscopy at ISOLDE, Phys. G: Nucl. Part. Phys. 44 (2017) 094004.

[16] O.J. Roberts, A.M. Bruce, P.H. Regan, Z. Podolyák, C.M. Townsley, J.F. Smith, K.F. Mulholland, A. Smith, A $\mathrm{LaBr}_{3}$ :Ce fast-timing array for DESPEC at FAIR, Nucl. Instrum. Methods Phys. Res. A 748 (2014) 91.

[17] V. Vedia, M. Carmona-Gallardo, L.M. Fraile, H. Mach, J.M. Udías, Performance evaluation of novel $\mathrm{LaBr}_{3}(\mathrm{Ce})$ scintillator geometries for fast-timing applications, Nucl. Instrum. Methods Phys. Res. A 857 (2017) 98-105.

[18] J.-M. Régis, M. Rudigier, J. Jolie, A. Blazhev, C. Fransen, G. Pascovici, N. Warr, The time-walk of analog constant fraction discriminators using very fast scintillator detectors with linear and non-linear energy response, Nucl. Instrum. Methods Phys. Res. A 684 (2012) 36. 
[19] V. Vedia, H. Mach, L.M. Fraile, J.M. Udías, S. Lalkovski, Enhanced time response of 1 -in. $\mathrm{LaBr}_{3}(\mathrm{Ce})$ crystals by leading edge and constant fraction techniques, Nucl. Instrum. Methods Phys. Res. A 795 (2015) 144.

[20] P.H. Regan, Precision measurement of sub-nanosecond lifetimes of excited nuclear states using fast-timing coincidences with $\mathrm{LaBr}_{3}(\mathrm{Ce})$ detectors, Radiat. Phys. Chem. 116 (2015) 38, Proceedings of the 9th International Topical Meeting on Industrial Radiation and Radioisotope Measurement Applications.

[21] B. Cederwall, et al., Technical Report for the Design, Construction and Commissioning of the DESPEC Germanium Array Spectrometer - DEGAS, FAIR Publications, 2014.

[22] V. Sánchez-Tembleque, V. Vedia, L.M. Fraile, S. Ritt, J.M. Udías, Optimizing time-pickup algorithms in radiation detectors with a genetic algorithm, Nucl. Instrum. Methods Phys. Res. A 927 (2019) 54-62.

[23] M. Nakhostin, Z. Podolyák, P.H. Regan, Digital processing of signals from $\mathrm{LaBr}_{3}$ :Ce scintillation detectors, J. Instrum. 9 (2014) C12049.

[24] E.R. Gamba, A Compton-background-correction method for fast-timing measurements using $\mathrm{LaBr}_{3}(\mathrm{Ce})$ detectors: The case of ${ }^{114} \mathrm{Pd}$, Ph.D. thesis, University of Brighton, 2019, unpublished.

[25] J. Adamczewski-Musch, N. Kurz, GSI Multi-Branch System Reference Manual, Vers. 6.3, GSI, Gesellschaft für Schwerionenforschung mbH, 2017.

[26] P. Moreira, J. Serrano, T. Wlostowski, P. Loschmidt, G. Gaderer, White rabbit: Sub-nanosecond timing distribution over ethernet, in: ISPCS, IEEE, 2009.

[27] V.F.E. Pucknell, MIDAS - Multi instance data acquisition system, 2017, http: //npg.dl.ac.uk/MIDAS/.

[28] M. Yamashita, Time dependence of rate dependent photomultiplier gain and its implications, Rev. Sci. Instrum. 51 (6) (1980) 768-775.

[29] C.C. Lo, B. Leskovar, Performance studies of high gain photomultiplier having Z-configuration of micro channel plates, IEEE Trans. Nucl. Sci. NS-28 659 (1981).

[30] K.S. Shah, et al., $\mathrm{LaBr}_{3}$ :Ce scintillators for gamma ray spectroscopy, IEEE Trans. Nucl. Sci. 50 (2003) 2410.

[31] J.-M. Régis, H. Mach, G.S. Simpson, J. Jolie, G. Pascovici, N. Saed-Samii, N. Warr, A. Bruce, J. Degenkolb, L.M. Fraile, C. Fransen, D.G. Ghita, S. Kisyov, U. Koester, A. Korgul, S. Lalkovski, N. Marginean, P. Mutti, B. Olaizola, Z. Podolyák, P.H. Regan, O.J. Roberts, M. Rudigier, L. Stroe, W. Urban, D. Wilmsen, The generalized centroid difference method for picosecond sensitive determination of lifetimes of nuclear excited states using large fast-timing arrays, Nucl. Instrum. Methods Phys. Res. A 726 (2013) 191-202.

[32] M. Rudigier, S. Lalkovski, E.R. Gamba, A.M. Bruce, Z. Podolyák, P.H. Regan, M. Carpenter, S. Zhu, et al., Fast timing measurement using an $\mathrm{LaBr}_{3}(\mathrm{Ce})$ scintillator detector array coupled with gammasphere, Act. Phys. Pol. B 48 (2017) 351.

[33] J.-M. Régis, G. Pascovici, J. Jolie, M. Rudigier, The mirror symmetric centroid difference method for picosecond lifetime measurements via $\gamma-\gamma$ coincidences using very fast $\operatorname{LaBr}_{3}(\mathrm{Ce})$ scintillator detectors, Nucl. Instrum. Methods Phys. Res. A 622 (1) (2010) 83-92.

[34] J.-M. Régis, A. Esmaylzadeh, J. Jolie, V. Karayonchev, L. Knafla, U. Köster, Y.H. Kim, E. Strub, $\gamma-\gamma$ Fast timing at X-ray energies and investigation on various timing deviations, Nucl. Instrum. Methods Phys. Res. A 955 (2020) 163258.

[35] M.J. Martin, Nuclear data sheets for A = 152, Nucl. Data Sheets 114 (11) (2013) 1497-1847.

[36] M.J. Mallaburn, B.S.N. Singh, D.M. Cullen, D. Hodge, M.J. Taylor, M.M. Giles, L. Barber, C.R. Nita, R.E. Mihai, C. Mihai, R. Marginean, N. Marginean, C.R. Nobs, E.R. Gamba, A.M. Bruce, C. Scholey, P. Rahkila, P.T. Greenlees, H. Badran, T. Grahn, O. Neuvonen, K. Auranen, F. Bisso, D.M. Cox, A. Herzá, R. Julin, J. Konki, A.K. Lightfoot, J. Pakarinen, P. Papadakis, J. Partanen, M. Sandzelius, J. Sarén, J. Sorri, S. Stolze, J. Uusitalo, P.H. Regan, Z. Podolyák, S. Lalkovski, J.F. Smith, M. Smolen, A time-of-flight correction procedure for fast-timing data of recoils with varying implantation positions at a spectrometer focal plane, Nucl. Instrum. Methods Phys. Res. A 933 (2019) 18-29.

[37] E. Clement, C. Michelagnoli, G. de France, H.J. Li, A. Lemasson, et al., Conceptual design of the AGATA $1 \pi$ array at GANIL, Nucl. Instrum. Methods Phys. Res. A 855 (2017) 1.

[38] M. Heine, S. Courtin, G. Fruet, D.G. Jenkins, D. Montanari, L. Morris, P.H. Regan, M. Rudigier, D. Symochko, Gamma efficiency simulations towards coincidence measurements for fusion cross sections, J. Phys. Conf. Ser. 763 (1) (2016) 012005 .

[39] M. Rudigier, R.L. Canavan, P.H. Regan, P.-A. Söderström, M. Lebois, J.N. Wilson, N. Jovancevic, S. Bottoni, M. Brunet, N. Cieplicka-Orynczakh, S. Courtin, D.T. Doherty, K. Hadynska-Kleka, M. Heine, W. Iskra, V. Karayonchev, A. Kennington, P. Koseoglou, G. Lotay, G. Lorusso, M. Nakhostin, C.R. Nita, S. Oberstedt, Z. Podolyák, L. Qie, J.-M. Régis, R. Shearman, P.M. Walker, W. Witt, Isomer spectroscopy and sub-nanosecond half-life determination in ${ }^{178} \mathrm{~W}$ using the NuBALL array, Acta Phys. Polon. B 50 (2019) 661.

[40] R.L. Canavan, M. Rudigier, P.H. Regan, M. Lebois, J.N. Wilson, N. Jovancevic, P.-A. Söderström, S.M. Collins, D. Thisse, J. Benito, S. Bottoni, M. Brunet, N. Cieplicka-Orynczak, S. Courtin, D.T. Doherty, L.M. Fraile, K. Hadynska-Klek, G. Häfner, M. Heine, L.W. Iskra, V. Karayonchev, A. Kennington, P. Koseoglou, G. Lotay, G. Lorusso, M. Nakhostin, C.R. Niță, S. Oberstedt, Z. Podolyák, L. Qi, J.-M. Régis, V. Sánchez-Tembleque, R. Shearman, V. Vedia, W. Witt, Halflife measurements in ${ }^{164,166} \mathrm{Dy}$ using $\gamma-\gamma$ fast-timing spectroscopy with the $v$-ball spectrometer, Phys. Rev. C 101 (2020) 024313.
[41] M. Rudigier, P.M. Walker, R.L. Canavan, Z. Podolyák, P.H. Regan, P.-A. Söderström, M. Lebois, J.N. Wilson, N. Jovancevic, A. Blazhev, J. Benito, S. Bottoni, M. Brunet, N. Cieplicka-Orynczak, S. Courtin, D. Doherty, L.M. Fraile, K. Hadynska-Klek, M. Heine, L.W. Iskra, J. Jolie, V. Karayonchev, A. Kennington, P. Koseoglou, G. Lotay, G. Lorusso, M. Nakhostin, C.R. Nita, S. Oberstedt, L. Qi, J.-M. Régis, V. Sánchez-Tembleque, R. Shearman, W. Witt, V. Vedia, K.O. Zell, Multi-quasiparticle sub-nanosecond isomers in ${ }^{178} \mathrm{~W}$, Phys. Lett. B 801 (2020) 135140.

[42] Z. Patel, F. Browne, A.M. Bruce, N. Chiga, R. Daido, et al., Commissioning of a $\mathrm{LaBr}_{3}(\mathrm{Ce})$ array with EURICA at RIBF, RIKEN Accel. Prog. Rep. 47 (2014) 13.

[43] F. Browne, A.M. Bruce, T. Sumikama, I. Nishizuka, S. Nishimura, P. Doornenbal, G. Lorusso, P.-A. Söderström, H. Watanabe, R. Daido, Z. Patel, S. Rice, L. Sinclair, J. Wu, Z.Y. Xu, A. Yagi, H. Baba, N. Chiga, R. Carroll, F. Didierjean, Y. Fang, N. Fukuda, G. Gey, E. Ideguchi, N. Inabe, T. Isobe, D. Kameda, I. Kojouharov, N. Kurz, T. Kubo, S. Lalkovski, Z. Li, R. Lozeva, H. Nishibata, A. Odahara, Z. Podolyák, P.H. Regan, O.J. Roberts, H. Sakurai, H. Schaffner, G.S. Simpson, H. Suzuki, H. Takeda, M. Tanaka, J. Taprogge, V. Werner, O. Wieland, Lifetime measurements of the first $2^{+}$states in ${ }^{104,106} \mathrm{Zr}$ : Evolution of ground-state deformations, Phys. Lett. B 750 (2015) 448-452.

[44] P. Koseoglou, V. Werner, N. Pietralla, S. Ilieva, T. Nikšić, D. Vretenar, P. Alexa, M. Thürauf, C. Bernards, A. Blanc, A.M. Bruce, R.B. Cakirli, N. Cooper, L.M, Fraile, G. de France, M. Jentschel, J. Jolie, U. Köster, W. Korten, T. Kröll, S. Lalkovski, H. Mach, N. Mărginean, P. Mutti, Z. Patel, V. Paziy, Z. Podolyák, P.H. Regan, J.-M. Régis, O.J. Roberts, N. Saed-Samii, G.S. Simpson, T. Soldner, C.A. Ur, W. Urban, D. Wilmsen, E. Wilson, Low- $Z$ boundary of the $n=88-90$ shape phase transition: ${ }^{148} \mathrm{Ce}$ near the critical point, Phys. Rev. C 101 (2020) 014303.

[45] J. Wiederhold, V. Werner, R. Kern, N. Pietralla, D. Bucurescu, R. Carroll, N. Cooper, T. Daniel, D. Filipescu, N. Florea, R.-B. Gerst, D. Ghita, L. Gurgi, J. Jolie, R.S. Ilieva, R. Lica, N. Marginean, R. Marginean, C. Mihai, I.O. Mitu, F. Naqvi, C. Nita, M. Rudigier, S. Stegemann, S. Pascu, P.H. Regan, Evolution of E2 strength in the rare-earth isotopes ${ }^{174,176,178,180} \mathrm{Hf}$, Phys. Rev. C 99 (2019) 024316.

[46] I.-Y. Lee, The gammasphere, Prog. Part. Nucl. Phys. 28 (1992) 473-485.

[47] J.T. Anderson, M. Albers, M. Alcorta, C. Campbell, M.P. Carpenter, C.J. Chiara, M. Cromaz, H.M. David, D. Doering, D.T. Doherty, C.R. Hoffman, R.V.F. Janssens, J. Joseph, T.L. Khoo, A. Kreps, T. Lauritsen, I.Y. Lee, C. Lionberger, C.J. Lister, T. Madden, M.B. Oberling, A.M. Rogers, D. Seweryniak, P. Wilt, S. Zhu, S. Zimmermann, A digital data acquisition system for the detectors at Gammasphere, in: 2012 IEEE Nuclear Science Symposium and Medical Imaging Conference Record (NSS/MIC), 2012, pp. 1536-1540.

[48] E.R. Gamba, A.M. Bruce, M. Rudigier, Treatment of background in $\gamma-\gamma$ fast-timing measurements, NIMA 928 (2019) 93.

[49] G. Fernandez-Martinez, Measurement of the Lifetimes of Excited States in Neutron-Rich Ce Isotopes, Ph.D. thesis, TU Darmstadt, 2018.

[50] E.R. Gamba, S. Lalkovski, M. Rudigier, A.M. Bruce, S. Bottoni, M.P. Carpenter, S. Zhu, A.D. Ayangeakaa, J.T. Anderson, T.A. Berry, I. Burrows, R.J. Carroll, P. Copp, M. Carmona-Gallardo, D.M. Cullen, T. Daniel, J.P. Greene, L.A. Gurgi, D.J. Hartley, R. Ilieva, S. Ilieva, R.V.F. Janssens, F.G. Kondev, T. Kroll, G.J. Lane, T. Lauritsen, I. Lazarus, G. Lotay, G. Fernandez-Martinez, Z. Podolyák, V.F.E. Pucknell, M. Reed, P.H. Regan, J. Rohrer, J. Sethi, D. Seweryniak, C.M. Shand, J. Simpson, M. Smolen, E.A. Stefanova, V. Vedia, O. Yordanov, Fasttiming measurements in neutron-rich odd-mass zirconium isotopes using $\mathrm{LaBr}_{3}$ :Ce detectors coupled with gammasphere, EPJ Web of Conf. 193 (2018) 05004.

[51] E.R. Gamba, A.M. Bruce, M. Rudigier, S. Lalkovski, S. Bottoni, M.P. Carpenter, S. Zhu, A.D. Ayangeakaa, J.T. Anderson, T.A. Berry, I. Burrows, R.J. Carrol, P. Copp, M. Carmona-Gallardo, D.M. Cullen, T. Daniel, G. Fernandez-Martinez, J.P. Greene, L.A. Gurgi, D.J. Hartley, R. Ilieva, S. Ilieva, R.V.F. Janssens, F.G. Kondev, T. Kroll, G.J. Lane, T. Lauritsen, I. Lazarus, G. Lotay, C.R. Nita, Z. Podolyák, V.F.E. Pucknell, M. Reed, P.H. Regan, J. Rohrer, J. Sethi, D. Seweryniak, C.M. Shand, J. Simpson, M. Smolen, V. Vedia, E.A. Stefanova, O. Yordanov, Fast-timing measurements in ${ }^{100} \mathrm{Zr}$ using $\mathrm{LaBr}_{3}(\mathrm{Ce})$ detectors coupled with gammasphere, Acta Phys. Polon. B 49 (2018) 555.

[52] E.R. Gamba, A.M. Bruce, S. Lalkovski, M. Rudigier, S. Bottoni, M.P. Carpenter, S. Zhu, J.T. Anderson, A.D. Ayangeakaa, T.A. Berry, I. Burrows, M.C. Gallardo, R.J. Carroll, P. Copp, D.M. Cullen, T. Daniel, G.F. Martínez, J.P. Greene, L.A. Gurgi, D.J. Hartley, R. Ilieva, S. Ilieva, F.G. Kondev, T. Kröll, G.J. Lane, T. Lauritsen, I. Lazarus, G. Lotay, C.R. Nita, Z. Podolyák, V. Pucknell, M. Reed, P.H. Regan, J. Rohrer, J. Sethi, D. Seweryniak, C.M. Shand, J. Simpson, M. Smoleń, E.A. Stefanova, V. Vedia, O. Yordanov, Fast-timing measurements in the ground-state band of ${ }^{114} \mathrm{Pd}$, Phys. Rev. C 100 (2019) 044309.

[53] J.T. Anderson, MRIAD user manual, 2015, Argonne National Lab Report ANL-15/07.

[54] S. Ansari, J.-M. Régis, J. Jolie, N. Saed-Samii, N. Warr, W. Korten, M. Zielińska, M.-D. Salsac, A. Blanc, M. Jentschel, U. Köster, P. Mutti, T. Soldner, G.S. Simpson, F. Drouet, A. Vancraeyenest, G. de France, E. Clément, O. Stezowski, C.A. Ur, W. Urban, P.H. Regan, Z. Podolyák, C. Larijani, C. Townsley, R. Carroll, E. Wilson, H. Mach, L.M. Fraile, V. Paziy, B. Olaizola, V. Vedia, A.M. Bruce, O.J. Roberts, J.F. Smith, M. Scheck, T. Kröll, A.-L. Hartig, A. Ignatov, S. Ilieva, S. Lalkovski, N. Mărginean, T. Otsuka, N. Shimizu, T. Togashi, Y. Tsunoda, Experimental study of the lifetime and phase transition in neutron-rich ${ }^{98,100,102} \mathrm{Zr}$, Phys. Rev. C 96 (2017) 054323. 
[55] A.G. Smith, R.M. Wall, D. Patel, G.S. Simpson, D.M. Cullen, J.L. Durell, S.J. Freeman, J.C. Lisle, J.F. Smith, B.J. Varley, G. Barreau, M. Petit, C. Theisen, E. Bouchez, M. Houry, R. Lucas, B. Cahan, A.L. Coguie, B.J.P. Gall, O. Dorvaux, N. Schulz, Lifetimes of yrast rotational states of the fission fragments $100 \mathrm{Zr}$ and 104Mo measured using a differential plunger, J. Phys. G: Nucl. Part. Phys. 28 (8) (2002) 2307-2316.

[56] G. Fruet, et al., Advances in the direct study of carbon burning in massive stars, Phys. Rev. Lett. (2020) in press.

[57] M. Heine, S. Courtin, G. Fruet, D.G. Jenkins, L. Morris, D. Montanari, M. Rudigier, P. Adsley, D. Curien, S. Della Negra, J. Lesrei, C. Beck, The STELLA apparatus for particle-Gamma coincidence fusion measurements with nanosecond timing, Nucl. Instrum. Methods Phys. Res. A 903 (2018) 1-7.
[58] G. Fruet, S. Courtin, D.G. Jenkins, M. Heine, D. Montanari, L.G. Morris, P. Adsley, C. Beck, S.D. Negra, F. Haas, F. Hammache, O.S. Kirsebom, A. Meyer, P.H. Regan, M. Rudiger, N. Séréville, C. Stodel, Fusion cross section measurements of astrophysical interest for light heavyions systems within the STELLA project, EPJ Web Conf. 163 (2019) 00018.

[59] M. Rejmund, B. Lecornu, A. Navin, C. Schmitt, S. Damoy, O. Delaune, J. Enguerrand, G. Fremont, P. Gangnant, L. Gaudefroy, B. Jacquot, J. Pancin, S. Pullanhiotan, C. Spitaels, Performance of the improved larger acceptance spectrometer: VAMOS++, Nucl. Instrum. Methods Phys. Res. A 646 (1) (2011) 184-191, http://dx.doi.org/10.1016/j.nima.2011.05.007, URL http: //www.sciencedirect.com/science/article/pii/S0168900211008515.

[60] L.M. Fraile, H. Mach, V. Vedia, B. Olaizola, V. Paziy, E. Picado, J.M. Udías, Fast timing study of a $\mathrm{CeBr}_{3}$ crystal: Time resolution below 120 ps at ${ }^{60} \mathrm{Co}$ energies, Nucl. Instrum. Methods Phys. Res. A 701 (2013) 235-242. 